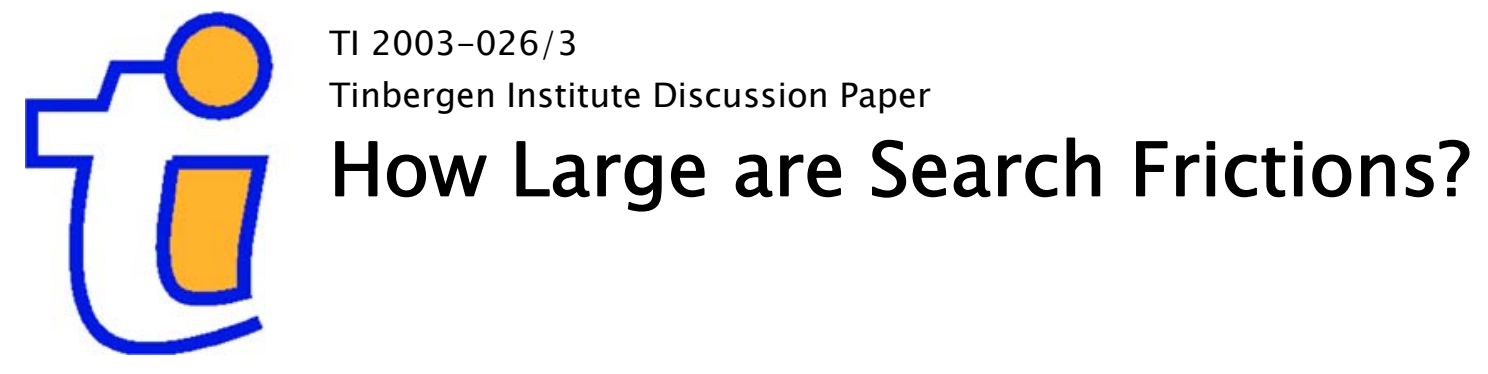

To appear in The Journal of the European Economic Association

Pieter A. Gautier 1,3

Coen N. Teulings 2,3

1 Vrije Universiteit Amsterdam, 2 Universiteit van Amsterdam, ${ }^{3}$ Tinbergen Institute, 


\section{Tinbergen Institute}

The Tinbergen Institute is the institute for economic research of the Erasmus Universiteit Rotterdam, Universiteit van Amsterdam, and Vrije Universiteit Amsterdam.

Tinbergen Institute Amsterdam

Roetersstraat 31

1018 WB Amsterdam

The Netherlands

Tel.: $\quad+31(0) 205513500$

Fax: $\quad+31(0) 205513555$

Tinbergen Institute Rotterdam

Burg. Oudlaan 50

3062 PA Rotterdam

The Netherlands

Tel.: $\quad+31(0) 104088900$

Fax: $\quad+31(0) 104089031$

Please send questions and/or remarks of nonscientific nature to driessen@tinbergen.nl.

Most TI discussion papers can be downloaded at http://www.tinbergen.nl. 


\title{
How Large are Search Frictions? *
}

\author{
Pieter A. Gautier ${ }^{\dagger}$ \\ Coen N. Teulings $\ddagger$
}

July 15, 2005

\begin{abstract}
This paper shows that we can normalize job and worker characteristics such that without frictions there exists a linear relationship between wages on the hand and worker and job type indices on the other hand. However, for 5 European countries and the US we find strong evidence for a systematic concave relation. An assignment model with search frictions provides a parsimonious explanation for our findings. This model yields two restrictions on the coefficients which fit the data well. Allowing for unobserved heterogeneity and measurement error we find that reservation wages are $25 \%$ lower than they would be in a frictionless world. Our results relate to the literature on industry wage differentials and on structural identification in hedonic models.
\end{abstract}

Keywords: wage differentials, search, assignment

JEL codes: J210, J300, J600, J230

${ }^{*}$ We thank the editor Orazio Attanasio, 3 anonymus referees, Gadi Barlevy, James Heckman, Dale Mortensen, Robert Shimer and seminar participants at the University of Chicago, the IZA in Bonn, Pompeu Fabra Barcelona, and the SED 2004 in Paris for useful comments and suggestions. We thank Miguel Portela for the Portugal estimates.

${ }^{\dagger}$ Erasmus University Rotterdam, Vrije Universiteit Amsterdam and Tinbergen Institute Amsterdam; gautier@tinbergen.nl

$\ddagger$ University of Amsterdam, SEO economic research, and Tinbergen Institute; teulings@seo.eur.nl 


\section{Introduction}

How much output is lost due to search frictions? To what extent do wages deviate from the simple Walrasian rule of "one good, one price", implying that workers with equal human capital should earn equal wages? Labor economist have been struggling with these questions for ages. Empirical inference is troubled by the fact that residuals in simple Mincer type earnings regressions can be due to at least three factors: (1) imperfect measurement of the relevant human capital variables, (2) measurement error in wages, and (3) non-Walrasian features of the labor market like search frictions, resulting in wage dispersion among otherwise homogeneous workers. A simple way to decompose the error term in these three components is not available.

This paper makes the following contributions. We show that if worker skill and job complexity can be described by one dimensional indices then there exists a normalization such that in a frictionless economy wages only depend linearly on these indices. If there are search frictions and wages are determined by Nash bargaining, then wages are concave in those indices. In practice, we typically do not observe all factors that are relevant for worker skill and job complexity and wages are measured with error. We show that our identification strategy still works if the third moments of the distributions of observable and unobservable worker and job characteristics are approximately equal to zero, as holds for the normal distribution. Even if this condition is not satisfied, only strange assumptions on these distributions can rationalize our empirical findings for the US and five European countries. Our results on the concavity of log wages are almost identical across the six countries that we consider but the required third moments that could rationalize our findings in the absence of search frictions would have to differ widely across these countries. The empirical evidence, and the regularity of our results across countries, provides strong support for the relevance of search frictions above unobserved heterogeneity.

The seminal papers by Dickens and Katz (1987) and Krueger and Summers (1988) started a debate on the nature of inter-industry wage differentials. Some contributors claimed that the industry effects in simple OLS earnings regressions are the reflection of genuine wage differentials between workers with equal human capital in different industries. These differentials might be driven by efficiency wages or rent sharing. Others took a more sceptical position, claiming that industry differentials might very well be at- 
tributed to unobserved worker characteristics which are correlated with industry choice, see Murphy and Topel (1987). The large literature on the measurement error in schooling variables, see Angrist and Krueger (1991), reveals that the importance of these unobserved characteristics should not be underestimated. Recent contributions apply matched worker-firm data to resolve the issue, see Abowd, Kramarz, and Margolis (1998).

Another strand in this literature starts from Burdett and Mortensen's (1998) model of monopsonistic wage setting in a world with on the job search. A high wage raises the inflow and reduces the outflow of workers, but it also reduces profits by increasing the wage bill. This trade-off results in a non-degenerate equilibrium wage distribution for workers with equal human capital. Larger firms pay higher wages and have longer average tenures. These correlations allow for inference on the dispersion of wages, holding constant the human capital of the worker, see Van den Berg and Ridder (1998) and Postel Vinay and Robin (2002).

This paper applies the search model of Teulings and Gautier (2004), that is based on the assignment models analyzed by Sattinger (1975) and Teulings (1995). Workers vary by their level of skill (or human capital) and jobs by their level of complexity. Both the skill and the complexity index vary continuously, so that there is an infinitum of job and worker types. Highly skilled workers are assumed to have an absolute advantage in all jobs and a comparative advantage in complex jobs. In the Walrasian version of this model, each worker type is assigned to a unique job type, where output reaches its maximum. Both this optimal complexity level and log wages are increasing in the skill type of the worker, the former due to comparative advantage, the latter due to absolute advantage. In the presence of search frictions, workers will not wait for ever till this unique first best job type comes along. When a contact occurs between a worker and a job, both face a trade off between either the pay off of matching with the partner that is available now or waiting for a more suitable partner. Hence, workers accept a range of job types, instead of a single job type as in the Walrasian equilibrium. Suppose that wages are set by Nash bargaining between the worker and the firm. Then, both sides share the loss in output relative to what it would be in the optimal assignment. Wages for a particular type of worker are concave in the job type: the wage reaches a maximum for the level of job complexity that maximizes output; it is lower for either less or more complex jobs.

Using standard human capital variables, we construct a worker skill index that we 
normalize such that it is linearly related to wages. Similarly, we construct a complexity index linearly related to wages, using industry and occupation dummies. When both indices enter jointly in a wage regression, their coefficients have no structural interpretation, since both indices are perfectly collinear in the Walrasian case, due to the comparative advantage assumption. Hence, the size of both coefficients is determined by the share of unobserved heterogeneity in the variance of both indices, and not by the underlying structure of the economy. ${ }^{1}$ We show, however, that it is difficult to justify the second order terms on these grounds. Our methodology allows a back-of-the-envelope calculation of the size of the cost of search, which is defined as the relative gap between the worker's reservation wage and the wage she would receive in a hypothetical Walrasian world. This cost of search is estimated to be between 15 and $30 \%$. The output losses due to non-Walrasian features of the labor market are therefore substantial.

The paper is organized as follows. Section 2 derives a theoretical relation between wages and worker and job characteristics in the presence of search frictions. This relation is used in Section 3 to explain why in a simple Mincerian type of wage equation, second order terms of worker skills and job characteristics are significant. We also discuss whether or not these second order terms can be interpreted in terms of unobserved characteristics. Section 4 concludes by relating our results to the literature on industry wage differentials and on structural identification of hedonic models.

\section{Wage formation in a world with search frictions}

\subsection{The Walrasian point of reference}

Consider a Walrasian world where workers and jobs are heterogeneous. Workers differ by their level of skill, denoted $\widehat{s}$, and jobs by their level of complexity, denoted $\widehat{c}$. Both indices vary continuously on the real domain. High skilled workers have an absolute advantage in all jobs and a comparative advantage in complex jobs. The main features of this type of world are discussed extensively in Teulings $(1995,2005)$, so we state the characteristics of market equilibrium without proof. Let $\widehat{y}(\widehat{s}, \widehat{c})$ denote the log market value of output of worker type $\widehat{s}$ at job type $\widehat{c}$. We assume $\widehat{y}(\cdot)$ to be twice differentiable in both its

\footnotetext{
${ }^{1}$ For the purpose of this paper, it is irrelevant whether there is measument error in observed characteristics or unobserved heterogeneity, because the former can always be respecified in terms of the latter. Hence, we discuss our results in terms of unobserved heterogeneity.
} 
arguments. Absolute advantage implies that better skilled workers are more productive than their colleagues with less skills in any job, $\widehat{y}_{\widehat{s}}(\widehat{s}, \widehat{c})>0$. Hence, better skilled workers earn higher wages in equilibrium. The Ricardian concept of comparative advantage implies that better skilled workers are relatively more productive in more complex jobs and leads to positive assortative matching (PAM). This requires productivity to be $\log$ supermodular in $\widehat{s}$ and $\widehat{c}$ : $\widehat{y}_{\widehat{s} \widehat{c}}(\widehat{s}, \widehat{c})>0 .^{2}$ Furthermore, we assume that $\widehat{y}(\widehat{s}, \widehat{c})$ has a unique interior maximum in $\widehat{c}$ for each $\widehat{s}$ and that a free entry-zero profit condition for firms applies. Hence, log wages are equal to the value of output. Finally, the value of leisure is zero, so that workers prefer working for any positive wage over non-employment.

In this Walrasian equilibrium, workers of type $\widehat{s}$ are assigned to that job type $\widehat{c}(\widehat{s})$ where they produce the highest value of output, and by implication, where they earn the highest wage. By construction, $\widehat{y}[\widehat{s}, \widehat{c}(\widehat{s})]$ satisfies the first and second order condition for a maximum:

$$
\begin{aligned}
\widehat{y} \widehat{c}[\widehat{s}, \widehat{c}(\widehat{s})] & =0 \\
\widehat{y}_{\widehat{c} \widehat{c}}[\widehat{s}, \widehat{c}(\widehat{s})] & <0
\end{aligned}
$$

since $\widehat{y}(\widehat{s}, \widehat{c})$ is differentiable and since there is an interior maximum of $\widehat{y}(\widehat{s}, \widehat{c})$ in $\widehat{c}$ for each $\widehat{s}$. Under the assumptions above, this equilibrium assignment $\widehat{c}(\widehat{s})$ is a differentiable function. It is strictly increasing, $\widehat{c}(\widehat{s})>0$, due to comparative advantage of better skilled workers in more complex jobs. ${ }^{3}$ Since $\widehat{c}(\widehat{s})$ is strictly increasing, it has a well defined inverse function, $\widehat{s}=\widehat{s}(\widehat{c}), \widehat{s}(\widehat{c})>0$, with $\widehat{s}[\widehat{c}(\widehat{s})]=\widehat{s}$. Hence, the equilibrium assignment is described by a one-to-one correspondence between $\widehat{s}$ and $\widehat{c}$. Each skill type is assigned to a unique job type and vice versa. The equilibrium locus of log wages $w$ is a twice differentiable function of the skill level $\widehat{s}, w=\widehat{w}^{*}(\widehat{s})=\widehat{y}^{*}(\widehat{s})$. It is strictly increasing, $\widehat{w}^{* \prime}(\widehat{s})>0$, due to absolute advantage of better skilled workers in any job type. The superscript $*$ indicates that $\widehat{w}^{*}(\widehat{s})$ is the log wage for worker type $\widehat{s}$ when assigned

\footnotetext{
${ }^{2}$ Since Becker's (1973) seminal paper on mariage markets, it has been standard to associate PAM with supermodularity. However, the Ricardian concept of comparative advantage, where the output of a match is tradable and there is free entry, requires log supermodularity, see Teulings and Gautier (2004) for a further analysis.

${ }^{3}$ Differentiating the identity: $\widehat{y}_{\widehat{c}}[\widehat{s}, \widehat{c}(\widehat{s})]=0$ with respect to $\widehat{s}$ yields:

$$
\widehat{y} \widehat{s c}[\widehat{s}, \widehat{c}(\widehat{s})]+\widehat{y} \widehat{c c}[\widehat{s}, \widehat{c}(\widehat{s})] \widehat{c}^{\prime}(\widehat{s})=0
$$

Comparative advantage implies $\widehat{y}_{\widehat{s} \widehat{c}}[\widehat{s}, \widehat{c}(\widehat{s})]>0$, the second order condition for a maximum implies $\widehat{y} \widehat{c c}[\widehat{s}, \widehat{c}(\widehat{s})]<0$. Hence: $\widehat{c}^{\prime}(\widehat{s})>0$.
} 
to her optimal job type $\widehat{c}(\widehat{s})$, and mutatis mutandis the same for $\widehat{y}^{*}(\widehat{s}) \equiv \widehat{y}[\widehat{s}, \widehat{c}(\widehat{s})]$. Combining these results yields $d \widehat{w}^{*}[\widehat{s}(\widehat{c})] / d \widehat{c}=\widehat{w}^{* \prime}[\widehat{s}(\widehat{c})] \widehat{s}^{\prime}(\widehat{c})>0$ : log wages can also be written as an increasing function of job complexity $\widehat{c}$. To summarize, log wages can be written as an increasing function of either the skill level $\widehat{s}$ and or the level of job complexity $\widehat{c}$, since the skill level $\widehat{s}$ is an increasing function of the complexity level $\widehat{c}$ (and vice versa).

We have not yet defined the units of measurement of $\widehat{s}$ and $\widehat{c}$. For the subsequent analysis, it is useful to apply a convenient normalization of both indices. Start with the the untransformed indices, $\hat{s}$ and $\hat{c}$. Without loss of generality we can choose the unit of measurement of wages such that $\mathrm{E}[w]=0$. Next, we can apply any increasing twice differentiable transformations to $\hat{s}$ and $\hat{c}$ without loosing any features of the equilibrium that we discussed above. Absolute and comparative advantage of better skilled workers, the one-to-one correspondence between $\hat{s}$ and $\hat{c}$, and the twice differentiability of the $\log$ wage function, all these features are invariant to such transformations. We define the transformed skill index $s$ such that: $s \equiv \hat{w}^{*}(\hat{s})$. After this transformation, the log wage in the optimal assignment is a linear function of the transformed skill variable, with a unit slope: $w^{*}(s)=s$, where we indicate that $w^{*}(\cdot)$ is a function of the transformed skill index $s$ rather than the untransformed $\widehat{s}$ by deleting its hat ${ }^{\wedge}$. This notation applies to all functions used throughout the paper: functions of the untransformed indices $\widehat{s}$ and $\widehat{c}$ have a hat $\left({ }^{\wedge}\right)$ on top, functions of the transformed indices $s$ and $c$ haven't. Because $\mathrm{E}[w]=0$, we have: $\mathrm{E}[s]=0$.

Since $\widehat{s}$ uniquely determines $w, w=\widehat{w}^{*}(\widehat{s})$. There is an alternative interpretation of this the transformation $\{\widehat{s}\} \rightarrow\{s\}$, namely: $s \equiv \mathrm{E}[w \mid \widehat{s}]$. Further note that $\mathrm{E}[w \mid \widehat{s}]$ is an increasing function of $\widehat{s}$ (since $\mathrm{E}[w \mid \widehat{s}]=\widehat{w}^{*}(\widehat{s})$ ), therefore the expectation operator uniquely defines $s$ as a transformation of $\widehat{s}$. While this interpretation is of little help in the Walrasian case, it will be of help in a world with search frictions, since then the one-to-one correspondence between $\widehat{s}$ and $w$ no longer applies.

Likewise, a convenient transformation of $\hat{c}$ is $c \equiv \widehat{w}^{*}[\hat{s}(\hat{c})]$. Again, we can also write: $c \equiv \mathrm{E}[w \mid \widehat{c}]$. Then, log wages in the optimal assignment are also a linear function of the transformed complexity variable, also with a unit slope, $w^{*}[s(c)]=c$, and hence: $\mathrm{E}[c]=0$. As a further consequence, the assignment of workers to jobs is linear with unit slope: $c(s)=s$ (or equivalently: $s(c)=c$ ). It is important to note that these normalizations 
can be imposed without loss of generality. Note furthermore that the only assumptions we have made are: the differentiability of the output function $y(\cdot)$, the existence of single dimensional worker and job type indices, absolute and comparative advantage of higher skilled types at more complex jobs, and the existence of an interior maximum in $c$.

\subsection{Adding search frictions}

The Walrasian assignment model of the previous section can be extended with search frictions, following the analysis of Teulings and Gautier (2004). The idea is that now workers meet only a limited number of job types per period and vice versa. For simplicity, we rule out on the job search: workers can only search while being unemployed. In the Walrasian economy, a worker of skill type $\widehat{s}$ is assigned to the unique job type $\widehat{c}(\widehat{s})$ that maximizes the value of her output, yielding a one-to-one correspondence between $\widehat{s}$ and $\widehat{c}$. Hence, both variables are perfectly correlated. In the presence of search frictions, this oneto-one correspondence breaks down, so that the correlation between $\widehat{s}$ and $\widehat{c}$ is no longer perfect. Workers cannot afford to wait for ever till the optimal job type $\widehat{c}(\widehat{s})$ comes along, and mutatis mutandis the same for firms. Hence, they accept a set of job types instead of just the optimal job. Let $\widehat{r}(\widehat{s})$ be the log reservation wage of the worker. Workers accept only jobs that pay a log wage of at least $\widehat{r}(\widehat{s})$. In the Walrasian equilibrium, this reservation wage is equal to the log wage and to log output: $\widehat{y}^{*}(\widehat{s})=\widehat{w}^{*}(\widehat{s})=\widehat{r}(\widehat{s})$, since workers will not accept any wage offer that pays less than what they can get in the optimal assignment. Search frictions make workers less choosy, since they cannot afford to search forever till they find the optimal job type. This reduces log reservation wages $\widehat{r}(\widehat{s})$ below $\widehat{y}^{*}(\widehat{s})$ :

$$
\widehat{r}(\widehat{s}) \equiv \widehat{y}^{*}(\widehat{s})-\widehat{x}(\widehat{s})=\widehat{y}[\widehat{s}, \widehat{c}(\widehat{s})]-\widehat{x}(\widehat{s})
$$

The variable $\widehat{x}(\widehat{s})$ has a nice interpretation. Let $\delta$ be the discount rate. Then, the asset value of an infinitely lived job seeker entering the Walrasian economy is $\frac{1}{\delta} \exp \left[\widehat{y}^{*}(\widehat{s})\right]$ : the worker immediately finds a job and collects her log wage $\widehat{w}^{*}(\widehat{s})=\widehat{y}^{*}(\widehat{s})$ each period; the asset value of employment is the net discounted value of all these wage payments. Likewise, the asset value of a job seeker entering the economy with search frictions is equal to the net discounted value of her reservation wage, $\frac{1}{\delta} \exp [\widehat{r}(\widehat{s})]$, she accepts any job offer that makes her better of than continued of job search. Moreover, she chooses her reservation wage such that it maximizes the asset value of job search. Hence, by construction, one minus the 
ratio of $\frac{1}{\delta} \exp [\widehat{r}(\widehat{s})]$ to $\frac{1}{\delta} \exp \left[\widehat{y}^{*}(\widehat{s})\right]$ measures the value loss due to search frictions relative to the asset value of being employment at the optimal assignment for a new participant in the labor market. This ratio is equal to $1-\exp \left[\widehat{r}(\widehat{s})-\widehat{y}^{*}(\widehat{s})\right]=1-\exp [-\widehat{x}(\widehat{s})]=$ $\widehat{x}(\widehat{s})+O\left[\widehat{x}(\widehat{s})^{2}\right]$. Hence, we refer to $\widehat{x}(\widehat{s})$ as the cost of search. The cost of search consists of three parts, the cost of maintaining vacancies, the output loss due to unemployment while seeking for a job, and the output loss due to a suboptimal assignment. ${ }^{4}$ Together, these three components add up exactly to the total cost of search $\widehat{x}(\widehat{s})$, see Teulings and Gautier (2004).

Search models imply that each match between a worker and a firm is characterized by a positive surplus $\widehat{y}(\widehat{s}, \widehat{c})-\widehat{r}(\widehat{s})$. We assume wages to be set by Nash bargaining over this surplus. Let $\beta$ be the worker's bargaining power parameter. Then, the log wage of an $\widehat{s}$-type worker in a $\widehat{c}$-type job satisfies approximately: ${ }^{5}$

$$
\widehat{w}(\widehat{s}, \widehat{c}) \cong \widehat{r}(\widehat{s})+\beta[\widehat{y}(\widehat{s}, \widehat{c})-\widehat{r}(\widehat{s})]
$$

Hence, $\widehat{w}(\widehat{s}, \widehat{c})$ is an increasing function of $\widehat{y}(\widehat{s}, \widehat{c})$ for a fixed $\widehat{s}$. Therefore, $\widehat{c}(\widehat{s})$ is also the value of $\widehat{c}$ that maximizes $\widehat{w}(\widehat{s}, \widehat{c})$ for a fixed $\widehat{s}: \widehat{w}[\widehat{s}, \widehat{c}(\widehat{s})] \equiv \widehat{w}^{*}(\widehat{s}){ }^{6}$

\footnotetext{
${ }^{4}$ Like in the Walrasian case, there are no other factors of production then labor. Firms have to pay a per period cost of maintaining a vacancy, which limits the supply of vacancies. Firms' expected share in the surplus compensates them for the cost of maintaining vacancies, see Teulings and Gautier (2004) for details.

${ }^{5}$ Strictly speaking, this relation applies in levels:

$$
W=R+\beta X
$$

However, for a small relative difference $x$, the difference is of higher order:

$$
w=r+\beta x+O\left[x^{2}\right]
$$

since $\ln (1+\beta x)=\beta x+O\left(x^{2}\right)$. Close to the Walrasian equilibrium, $x$ is small.

${ }^{6}$ There is an important difference between this model and Shimer and Smith (2000). They treat both sides of the market symmetrically, so that their wage equation reads:

$$
\widehat{w}(\widehat{s} \text { when matched to } \widehat{c})=\widehat{r}(\widehat{s})+\beta[\hat{y}(\widehat{s}, \widehat{c})-\widehat{r}(\widehat{s})-\widehat{r}(\widehat{c})]
$$

where by symmetry $\beta=\frac{1}{2}$. Hence, their $\hat{y}(\widehat{s}, \widehat{c})$ is not necessarily concave but $\hat{y}(\widehat{s}, \widehat{c})-\widehat{r}(\widehat{s})$ is localy concave as long as the matching set of $\widehat{s}$ has an interior upper and lower bound. Since there are no other factors of production than labor, the firm's outside option is zero in our model, so $\widehat{r}(\widehat{c})$ drops out. Hence, contrary to Shimer and Smith's model, the maximum of $y(\widehat{s}, \widehat{c})$ and $\widehat{w}(\widehat{s}, \widehat{c})$ for a given $\widehat{s}$ coincide.
} 
We normalize $\widehat{s}$ and $\widehat{c}$ along the lines we discussed in the previous section:

$$
\begin{aligned}
s & =\mathrm{E}[\widehat{w}(\widehat{s}, \widehat{c}) \mid \widehat{s}] \\
c & =\mathrm{E}[\widehat{w}(\widehat{s}, \widehat{c}) \mid \widehat{c}]
\end{aligned}
$$

These transformations require $\mathrm{E}[\widehat{w}(\widehat{s}, \widehat{c}) \mid \widehat{s}]$ and $\mathrm{E}[\widehat{w}(\widehat{s}, \widehat{c}) \mid \widehat{c}]$ to be increasing functions of $\widehat{s}$ and $\widehat{c}$ respectively. Without search frictions, this is always the case, see the previous section. With search frictions, it holds for all $\widehat{c}$, but there are some pathological cases, where this condition does not hold for all $\widehat{s}$. We rule out these cases by assumption. ${ }^{7}$.

The search equilibrium is depicted in Figure E. Panel A represents the Walrasian case, where $r(s)=w^{*}(s)=y^{*}(s)$, and hence $x(s)=0$. All workers of type $s$ are assigned to the job type $c(s)$ that maximizes their output. Panel B represents the case with search frictions: $r(s)$ is less than $y^{*}(s)$, the difference being $x(s)$. An $s$-type job seeker is less choosy than in a Walrasian world and accepts all $c$-type tasks for which $y(s, c) \geq r(s)$.

\footnotetext{
${ }^{7}$ Teulings and Gautier (2004) show that previous assumptions imply the matching set of type $\widehat{s}$ to be uniquely defined by a lower and upper bound, denoted $\widehat{c}^{-}(\widehat{s})$ and $\widehat{c}^{+}(\widehat{s})$, which are increasing in $\widehat{s}$. By Leibniz 'rule:

$$
\left.\frac{d \mathrm{E}[\widehat{w}(\widehat{s}, \widehat{c}) \mid \widehat{s}]}{d \widehat{s}}=\mathrm{E}\left[\widehat{w}_{\widehat{s}}(\widehat{s}, \widehat{c}) \mid \widehat{s}\right]+\frac{g\left[\widehat{c}^{+}(\widehat{s})\right] \widehat{c}^{+\prime}(\widehat{s})-g\left[\widehat{c}^{-}(\widehat{s})\right] \widehat{c}^{-\prime}(\widehat{s})}{G[\widehat{c}(\widehat{c}+\widehat{s})]-G\left[\widehat{c}^{-}(\widehat{s})\right]}-\mathrm{E}[\widehat{w}(\widehat{s}, \widehat{c}) \mid \widehat{s}]\right)
$$

where $g(\cdot), G(\cdot)$ denote the density and distribution function of type $\widehat{c}$. The first term is the wage change for intramarginal types $c$, which is always positive since $\widehat{w}_{\widehat{s}}(\widehat{s}, \widehat{c})>0$ by $\widehat{y}_{\widehat{s}}(\widehat{s}, \widehat{c})>0$. The second term measures the effect of changes in the composition of the matching set: jobs drop out at the lower bound, while new ones enter at the top. Both the new jobs and those which are dropping out pay less than the average one, since: $\widehat{r}(\widehat{s})<\mathrm{E}[\widehat{w}(\widehat{s}, \widehat{c}) \mid \widehat{s}]$. Hence, this second term is negative if: $g\left[\widehat{c}^{+}(\widehat{s})\right] \widehat{c}^{+\prime}(\widehat{s})>g\left[\widehat{c}^{-}(\widehat{s})\right] \widehat{c}^{-\prime}(\widehat{s})$. This term can dominate the first term, so that $d \mathrm{E}[\widehat{w}(\widehat{s}, \widehat{c}) \mid \widehat{s}] / d \widehat{s}$ can be negative.
}

For the derivative with respect to $\widehat{c}$, this problem does not occur:

$$
\begin{aligned}
\frac{d \mathrm{E}[\widehat{w}(\widehat{s}, \widehat{c}) \mid \widehat{c}]}{d \widehat{c}}= & \mathrm{E}\left[\widehat{w}_{\widehat{c}}(\widehat{s}, \widehat{c}) \mid \widehat{c}\right] \\
& +\frac{f\left[\widehat{s}^{+}(\widehat{c})\right] \widehat{s}^{+\prime}(\widehat{c})\left(\widehat{r}\left[\widehat{s}^{+}(\widehat{c})\right]-\mathrm{E}[\widehat{w}(\widehat{s}, \widehat{c}) \mid \widehat{c}]\right)-f\left[\widehat{s}^{-}(\widehat{c})\right] \widehat{s}^{-\prime}(\widehat{c})\left(\widehat{r}\left[\widehat{s}^{-}(\widehat{c})\right]-\mathrm{E}[\widehat{w}(\widehat{s}, \widehat{c}) \mid \widehat{c}]\right)}{F\left[\widehat{s}^{+}(\widehat{c})\right]-F\left[\widehat{s}^{-}(\widehat{c})\right]}
\end{aligned}
$$

where $f(\cdot)$ and $F(\cdot)$ denote density and distribution function of type $\widehat{s}$ and where $\widehat{s}^{-}(\widehat{c})$ and $\widehat{s}^{+}(\widehat{c})$ are the lower and upper bound of the matching set of type $\widehat{c}$. Since $\widehat{w}_{\widehat{s}}(\widehat{s}, \widehat{c})>0, \widehat{r}\left[\widehat{s}^{+}(\widehat{c})\right]=$ $\widehat{w}\left[\widehat{s}^{+}(\widehat{c}), \widehat{c}\right]>\mathrm{E}[\widehat{w}(\widehat{s}, \widehat{c}) \mid \widehat{c}]$ and likewise $\widehat{r}[\widehat{s}(\widehat{c})]<\mathrm{E}[\widehat{w}(\widehat{s}, \widehat{c}) \mid \widehat{c}]$. Hence, the second term is always positive. The Bellman equation for the asset value of a vacancy of type $\widehat{c}$ is of the form:

$$
\lambda=\left\{F\left[\widehat{s}^{+}(\widehat{c})\right]-F[\widehat{s}(\widehat{c})]\right\} \mathrm{E}[\widehat{w}(\widehat{s}, \widehat{c})-\widehat{r}(\widehat{s}) \mid \widehat{c}]
$$

where $\lambda$ is a function of the parameters of the model, see Teulings and Gautier (2004). This equation holds identically for all $\widehat{c}$. Hence, its first difference must also hold, implying: $\mathrm{E}\left[\widehat{w}_{\widehat{c}}(\widehat{s}, \widehat{c}) \mid \widehat{c}\right]=0$, and hence, $d \mathrm{E}[\widehat{w}(\widehat{s}, \widehat{c}) \mid \widehat{s}] / d \widehat{c}>0$. 
Log wages $w(s, c)$ are a weighted average of $r(s)$ and $y(s, c)$. Figure 1 depicts both situations in $s, c$-space: the Walrasian equilibrium is represented by the solid diagonal, the search equilibrium by the band around it.

Next, we simplify the analysis by the following assumption

Assumption 1: The cost of search are equal for all skill types: $x(s)=x$

By this assumption the cost of search is equal for all skill types, so that $x$ is the fraction of foregone output due to search frictions, i.e. the difference between output in a Walrasian economy and an economy with search frictions. In a more general model, Assumption 1 is likely to be violated. Here our aim is more modest. We are only interested in a first order approximation of $x(s)$. For that purpose, we ignore variations in $x(s)$ along the support of $s$. Teulings and Gautier (2004) derive an expression for $x(s)$ in terms of the primitives of the model. A suitable combination of the density of skill supply and the cost of maintaining vacancies, generates Assumption 1 as a result. Furthermore, we show that $x(s)$ is almost constant in simulations of the model for quite standard assumptions on these primitives, except for extreme values of $s$.

We apply a Taylor expansion around the Walrasian equilibrium, where the cost of search are zero, $x=0$. Let $\Delta c \equiv c-c(s)$ denote the deviation from the optimal allocation for type $s$ and let $\Delta c^{*} \equiv c^{+}(s)-c(s)$, where $c^{+}(s)$ is the most complex job that a worker of type $s$ is willing to accept, that is, for which $y(s, c) \geq r(s)$. In the Walrasian equilibrium, $\Delta c^{*}=0$. A worker of type $s$ turns down more complex jobs because the wage would be below her reservation wage. Nash bargaining implies that output is equal to the reservation wage at the boundary of the matching set. Hence:

$$
y\left[s, c(s)+\Delta c^{*}\right]=r(s)
$$

By a second order Taylor expansion of equation (1) with respect to $c$ around $c=c(s)$ and using $y_{c}[s, c(s)]=0$, we have:

$$
x \cong-\frac{1}{2} y_{c c} \Delta c^{*^{2}}
$$

We can make exactly the same argument for the least complex job that a worker of type $s$ is willing to accept. Hence, job offers with $|\Delta c|>\Delta c^{*}$ are rejected.

\section{Lemma 1:}


Up to a second order Taylor expansion, the matching set of a type s worker is symmetric around the midpoint $c(s)$, its upper bound being $c(s)+\Delta c^{*}$ and its lower bound being $c(s)-\Delta c^{*}$.

This lemma follows from the fact that a second order approximation of $y(s, c)$ around its maximum is a parabola which is always symmetric around its maximum. Lemma 1 can be used for the derivation of the expectation of $\Delta c^{2}$ in the matching set, again using a Taylor expansion: ${ }^{8}$

$$
\begin{aligned}
\mathrm{E}[\Delta c \mid s] & \cong 0 \\
\mathrm{E}\left[\Delta c^{2} \mid s\right] & \cong \frac{1}{3} \Delta c^{*^{2}} \cong-\frac{2}{3} y_{c c}^{-1} x
\end{aligned}
$$

The parameter $\left|y_{c c}\right|$ has a special interpretation. It is the curvature of the log cost function of a firm producing task type $c$, or, the complexity dispersion parameter, see Teulings (2005), measuring the productivity loss due to suboptimal assignments:

$$
\operatorname{Loss}(\Delta c)=y^{*}(s)-y[s, c(s)+\Delta c] \cong-\frac{1}{2} y_{c c} \Delta c^{2}
$$

where the second equality follows from the same Taylor expansion as in equation (3). Since the wage of the least attractive job type in the matching set of a worker of type $s$ is equal to her reservation wage, $\operatorname{Loss}\left(\Delta c^{*}\right)$ is equal to $x$. Consider a second order Taylor expansion of equation (2) around $w[s, c(s)]$ and a first order expansion of the expectation of $s$ conditional on $c$ and vice versa:

$$
\begin{aligned}
w(s, c) & \cong w_{0}+w_{s} s+w_{c} c+\frac{1}{2} w_{s s} s^{2}+w_{s c} s c+\frac{1}{2} w_{c c} c^{2} \\
w_{c c} & =\beta y_{c c}<0 \\
\mathrm{E}[c \mid s] & \cong \rho_{0}+\rho s \\
\mathrm{E}[s \mid c] & \cong \tau_{0}+\tau c
\end{aligned}
$$

\footnotetext{
${ }^{8}$ Let $g(\Delta c)=g_{0}+g_{1} \Delta c+O\left(\Delta c^{2}\right)$ be a second order Taylor expansion of the density function $\Delta c$ in the pool of vacancies. Then:

$$
\mathrm{E}\left[\Delta c^{2}\right]=\frac{\int_{-\Delta c^{*}}^{\Delta c^{*}} g(v) v^{2} d v}{\int_{-\Delta c^{*}}^{\Delta c^{*}} g(v) d v}=\frac{\frac{2}{3} g_{0} \Delta c^{*^{3}}+O\left(\Delta c^{*^{4}}\right)}{2 g_{0} \Delta c^{*}+O\left(\Delta c^{*^{2}}\right)}=\frac{1}{3} \Delta c^{*^{2}}+O\left(\Delta c^{*^{3}}\right)
$$$$
\mathrm{E}[\Delta c]=\frac{\int_{-\Delta c^{*}}^{\Delta c^{*}} g(v) v d v}{\int_{-\Delta c^{*}}^{\Delta c^{*}} g(v) d v}=\frac{\frac{2}{3} g_{1} \Delta c^{*^{3}}+O\left(\Delta c^{*^{4}}\right)}{2 g_{0} \Delta c^{*}+O\left(\Delta c^{*^{2}}\right)}=O\left(\Delta c^{*^{2}}\right)
$$ 
The inequality in the second line follows from the concavity of $y(s, c)$ in $c$. Note that the second order expansion implies the implicit assumption that the complexity dispersion parameter is constant along the domain of $s$. Variation in $y_{c c}$ along the domain of $s$ is a higher order phenomenon that will be ignored in the subsequent analysis. Likewise we can ignore variation in the cost of search $x$. The subsequent proposition relates the other partial derivatives of $w(s, c)$ to the parameters of the joint distribution of $s$ and $c$.

Proposition 1 Normalize $w, s$ and $c$ such that $E[w]=0, E[w(s, c) \mid s]=s$ and $E[w(s, c) \mid c]=$ c. Then, the Taylor expansions in equation (5) imply:

$$
w(s, c) \cong w_{0}+s-\omega\left[\rho s^{2}-(1+\rho) s c+c^{2}\right]
$$

where $\omega \equiv-\frac{1}{2} w_{c c}=-\frac{1}{2} \beta y_{c c}>0$ and $\sqrt{\rho}=\operatorname{Cor}[s, c]$ and where $w_{0}$ is an appropriate constant.

The proof is in Appendix A. Proposition 1 provides a simple economic theory for a wage function that is concave in $s$ and $c$. In the Walrasian equilibrium, $s$ and $c$ are perfectly correlated with $c=s$, and hence $\operatorname{Var}[c]=\operatorname{Var}[s]$. This equality does not hold in a search equilibrium. We show in Appendix A that $\operatorname{Var}[c]=\rho \operatorname{Var}[s]$, which is smaller than $\operatorname{Var}[s]$ since $\sqrt{\rho}$ is a correlation. This is due to absolute advantage: in the optimal assignment $c(s)$, a variation in $s$ has a first order effect in $y(s, c)$ (and hence on $w(s, c)$ ), while a variation in $c$ has only a second order effect on $y(s, c)$, since by construction $y_{c}[s, c(s)]=0$. Hence, comparing the two single variable regressions, the regression on $s$ "explains" a larger share of the variance of $w(s, c)$ than the one on $c$. Since we have normalized $\mathrm{E}[w(s, c) \mid s]=s$ and $\mathrm{E}[w(s, c) \mid c]=c$, the variance of $s$ must be larger than that of $c$.

The proof of Proposition 1 implies:

$$
\mathrm{E}\left[\Delta c^{2} \mid s\right] \cong \rho(1-\rho) \sigma^{2}
$$

where $\sigma^{2} \equiv \operatorname{Var}[s]$, see Appendix A. Substitution of these results in equation (3) yields a simple expression for $x$ :

$$
x=-\frac{3}{2} y_{c c} \rho(1-\rho) \sigma^{2}=3 \frac{\omega}{\beta} \rho(1-\rho) \sigma^{2}
$$


The variance of $w$ satisfies, see Appendix A:

$$
\begin{aligned}
\operatorname{Var}[w] & \cong \sigma^{2}+(1-\rho)^{2}(1+\rho) \rho \omega^{2} \sigma^{4} \\
& \cong \sigma^{2}+\frac{1+\rho}{9 \rho} \beta^{2} x^{2} \leq \sigma^{2}+\frac{1}{12} x^{2} \cong \sigma^{2}
\end{aligned}
$$

where the second step uses (7). The inequality applies if $\rho \geq 1 / 2$ and $\beta \leq 1 / 2$ which is reasonable from an empirical point of view, see e.g. Abowd and Lemieux (1993). The latter approximation shows that the variance of the second order term is small relative to the first order term for values of $x$ up to 0.50 .

Summing up, we have extended the assignment model of the previous section with a simple model of search frictions with Nash bargaining over wages. In this world, wages are a concave function of appropriately transformed worker and job characteristics. A second order Taylor expansion of this wage function allows us to characterize the relation between this wage function, the joint distribution of $s$ and $c$, and the cost of search $x$. We need only two additional assumptions on functional forms to derive these relations: both the cost of search $x$ and the complexity dispersion parameter $y_{c c}$ must be constant along the support of $s$. Since we focus on a second order approximation of the effect of search frictions, those assumptions are not restrictive because a violation of them has only higher order effects on $y(s, c)$.

\section{Empirical analysis}

\subsection{The measurement of the key variables}

A fundamental problem in the empirical analysis of non-Walrasian features of wages and worker-to-job assignments is the difficulty to distinguish between deviations from the frictionless assignment and measurement error in the data. Hence, if we want to apply the framework developed above for an empirical analysis, we have to allow for the fact that the three main ingredients of our analysis are all observed with a fair amount of unobserved heterogeneity or measurement error. Let $q$ be a vector of observed worker characteristics, and $m$ a vector of observed job characteristics. Without loss of generality, we can normalize all observed and unobserved characteristics such that they have a zero mean. Further, denote the observed skill and complexity indices by $\bar{s}$ and $\bar{c}$ respectively, the unobserved components by $\varepsilon_{s}$ and $\varepsilon_{c}$ and measurement error in wages by $\varepsilon_{w}$. Then, 
we can model, measurement error and unobserved heterogeneity as follows:

$$
\begin{aligned}
\bar{s} & \equiv \mathrm{E}[s \mid q] \\
\bar{c} & \equiv \mathrm{E}[c \mid m] \\
\varepsilon_{s} & \equiv s-\bar{s} \\
\varepsilon_{c} & \equiv c-\bar{c} \\
w & =w(s, c)+\varepsilon_{w}
\end{aligned}
$$

where $w(s, c)$ is the observed log wage, $\mathrm{E}\left[\varepsilon_{s}\right]=\mathrm{E}\left[\varepsilon_{c}\right]=\mathrm{E}\left[\varepsilon_{w}\right]=0$ and $\mathrm{E}\left[\bar{s} \varepsilon_{s}\right]=\mathrm{E}\left[\bar{c} \varepsilon_{c}\right]=\mathrm{E}\left[w \varepsilon_{w}\right]=$ $E\left[\varepsilon_{s} \varepsilon_{w}\right]=E\left[\varepsilon_{c} \varepsilon_{w}\right]=0$. Both the skill index $s$ and the complexity index $c$ are decomposed into two orthogonal parts. The measurement error in wages is uncorrelated with any other random variable in the model. Bound and Krueger (1991) report that the ratio of variance of the signal to the total variance in log hourly wages is 0.84 for the CPS. Given those definitions, it is not very restrictive to write,

$$
\begin{aligned}
& s=\chi^{\prime} q+\varepsilon_{s} \equiv \bar{s}+\varepsilon_{s} \\
& c=\gamma^{\prime} m+\varepsilon_{c} \equiv \bar{c}+\varepsilon_{c},
\end{aligned}
$$

since we can include everything and its square in $q$ and $m$. Therefore, any differentiable non-linear relation can be captured up to an arbitrary small degree of misspecification. Similarly, the additive separability between the observed and the unobserved component is not a restriction: it just implies that we define $\bar{c} \equiv \mathrm{E}[c \mid m]$ and $\varepsilon_{c}$ as the variation in $c$ orthogonal on $\mathrm{E}[c \mid q]$ and the same for $s$. Finally, note that we do not need to assume anything on $\mathrm{E}\left[\bar{c} \varepsilon_{s}\right]$ and $\mathrm{E}\left[\bar{s} \varepsilon_{c}\right]$.

\subsection{The estimation of $\bar{s}$ and $\bar{c}$}

How can we estimate the parameter vectors $\chi$ and $\gamma$ and hence the observed part of the skill and complexity indices, $\bar{s}$ and $\bar{c}$ ? If the real world is described by the Walrasian model of Section 2.1, then the answer is simple. We can simply apply OLS to the relations $w^{*}(s)$ and $w^{*}[s(c)]$ which directly relate wages to observed worker and job characteristics. Hence, in that case $\chi$ and $\gamma$ can be consistently estimated from the following regression models:

$$
\begin{aligned}
& w(s, c)=\chi^{\prime} q+\underline{\varepsilon}_{s} \\
& w(s, c)=\gamma^{\prime} m+\underline{\varepsilon}_{c}
\end{aligned}
$$


where $\underline{\varepsilon}_{s}$ and $\underline{\varepsilon}_{c}$ are error terms. The error terms in both regressions reflect the unobserved part of worker and job characteristics and the measurement error in log wages, that is: $\underline{\varepsilon}_{s}=\varepsilon_{s}+\underline{u}$ and $\underline{\varepsilon}_{c}=\varepsilon_{c}+\underline{u}$. Note that we run separate regressions for the supply and the demand side of the market. If we had included $q$ and $m$ simultaneously, it would be unclear whether our estimates reflect a supply or a demand side relationship; $q$ would have served partly as a proxy for the unobserved part in the complexity index, $\varepsilon_{c}$, and $m$ would have served partly as a proxy for the unobserved part in the skill index, $\varepsilon_{s}$. Since $s$ and $c$ are perfectly correlated, it is quite likely that $\bar{s}$ is correlated with $\varepsilon_{c}$ and that $\bar{c}$ is correlated with $\varepsilon_{s}$. Only by estimating both relations separately, we can give a structural interpretation to the regression coefficients. In fact, this procedure is similar to the approach proposed by Rosen (1974). We return to this issue below.

The simple procedure laid out in equation (10) works fine in a Walrasian world. However, if the real world is characterized by search frictions and if log wages therefore satisfy the concave function (6), then at first sight, this procedure does not seem to work anymore. Equation (6) includes quadratic terms in $s$ and $c$, so that estimates of $\chi$ and $\gamma$ that do not allow for this non-linearity seem to be biased. The subsequent proposition shows this intuition to be false:

Proposition 2 If (6) holds and measurement error in $s, c$ and $w$ is as in (9), then $\chi$ and $\gamma$ are consistently estimated by equation (10).

Proof. Equation (6) is constructed such that

$$
\begin{aligned}
& \mathrm{E}[w(s, c) \mid s]=s \\
& \mathrm{E}[w(s, c) \mid c]=c
\end{aligned}
$$

Since $\mathrm{E}[s \mid \bar{s}]=\bar{s}$ and $\mathrm{E}[c \mid \bar{c}]=\bar{c}$ and since $\underline{u}$ is uncorrelated to anything else, these equations imply $\mathrm{E}[w \mid \bar{s}]=\bar{s}$ and $\mathrm{E}[w \mid \bar{c}]=\bar{c}$. Hence, equation (10) gives consistent estimates of $\chi$ and $\gamma$.

The intuition for Proposition 2 is that the correlation between $w$ and $s$ introduced in equation (6) by the term $-\omega \rho s^{2}$ is exactly offset by the correlations introduced by both other second order terms, $\omega(1+\rho) s c$ and $-\omega c^{2}$, since $s$ and $c$ are positively correlated. Taking these three terms together, $w$ and $s^{2}$ are uncorrelated. Mutatis mutandis the same analysis applies to the correlation between $w$ and $c^{2}$. Apart from the unobserved 
heterogeneity in $s$ and $c$ respectively and the measurement error in log wages, the error terms $\underline{\varepsilon}_{s}$ and $\underline{\varepsilon}_{c}$ also capture the effect of suboptimal assignment due to search frictions. Proposition 2 is just a reflection of the way we have constructed our search model. We derived partial derivatives of $w(s, c)$ by imposing the restrictions $\mathrm{E}[w(s, c) \mid s]=s$ and $\mathrm{E}[w(s, c) \mid c]=c$. There is no loss of generality involved in imposing these restrictions, they just apply a proper scaling on the indices $s$ and $c$, see the discussion in Section 2.2. Proposition 2 implies that $w$ and $\bar{s}^{2}$ should be uncorrelated. This implication is imposed in our estimation procedure. We apply an iterative procedure such that if we enter both $\bar{s}$ and $\bar{s}^{2}$ in regression (10), then the coefficient on the second order term $\bar{s}^{2}$ is indeed exactly zero. First, we run the regression: $w=\chi_{1} \bar{s}_{1}+\chi_{2} \bar{s}_{1}^{2}+\underline{\varepsilon}_{s}$, where $\bar{s}_{1}$ is $E(s \mid q)$, constructed from equation (9) and $\varepsilon_{s_{1}}=s-\bar{s}_{1}$. Second, we construct a new variable $\bar{s}_{2}=\chi_{1} \bar{s}_{1}+\chi_{2} \bar{s}_{1}^{2}-\mathrm{E}\left[\chi_{1} \bar{s}_{1}+\chi_{2} \bar{s}_{1}^{2}\right]$ and rerun the first regression where we replace $\bar{s}_{1}$ by $\bar{s}_{2}$. We repeat these steps till $\chi_{2}=0$. Mutatis mutandis the same applies to our regression for $\bar{c} .^{9}$ This algorithm therefore normalizes $\bar{s}$ such that any correlation between $\bar{s}$ and $\varepsilon_{s}$ is eliminated.

Our empirical analysis for the United States applies the CPS March supplements for 1989-92. We consider full time, non-farmer, private sector workers aged between 16 and 65, which yields 222179 observations. We constructed hourly wages. The vector $q$ includes the usual variables: total years of schooling, a third order polynomial in experience, highest completed education, being married, having a full or part time contract including various cross terms of experience, education and being married; $m$ contains 520 occupation and 242 industry dummies. Besides $q$ and $m$, we add calendar time dummies to capture the effect of inflation and the business cycle. Obviously, these time dummies are not included in the construction of $\bar{s}$ and $\bar{c}$. Let $R_{w s}^{2}$ and $R_{w c}^{2}$ denote the $R^{2}$ statistics for both regressions (10); $R_{w s}^{2}=0.3358$ and $R_{w c}^{2}=0.3632$. Hence, the observed part of the skill and complexity indices capture a reasonable part of the total variance in log wages.

\footnotetext{
${ }^{9} 9$ iterations were sufficient for both $s$ and $c$.
} 


\subsection{Interpreting regressions with both $\bar{s}$ and $\bar{c}$}

When we enter $\bar{s}$ and $\bar{c}$ in an OLS regression on log wages simultaneously, we obtain the following results ( $\mathrm{t}$-values between brackets):

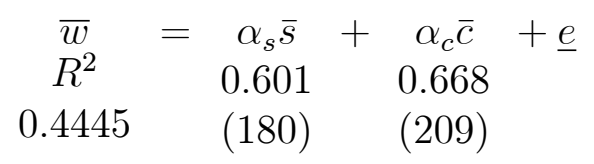

Can we give a structural interpretation to these coefficients? In Appendix B, we prove the following result:

Proposition 3 Consider the Walrasian assignment model $w^{*}(s)=s$ and $w^{*}[s(c)]=c$, the measurement model in equation (9), and the regression equation (11). The coefficients $\alpha_{s}$ and $\alpha_{c}$ satisfy:

$$
\left[\begin{array}{c}
\alpha_{s} \\
\alpha_{c}
\end{array}\right]=\frac{1}{R_{\bar{s}}^{2} R_{\bar{c}}^{2}-C^{2}}\left[\begin{array}{l}
R_{\bar{c}}^{2}\left(R_{\bar{s}}^{2}-C\right) \\
R_{\bar{s}}^{2}\left(R_{\bar{c}}^{2}-C\right)
\end{array}\right]
$$

where $R_{\bar{s}}^{2}$ and $R_{\bar{c}}^{2}$ are the share of the variances of $\bar{s}$ and $\bar{c}$ in the total variances of $s$ and c respectively, and where $C \equiv \operatorname{Corr}[\bar{s}, \bar{c}]$.

The proof follows directly from the formulas for OLS regression coefficients. We cannot identify $R_{\bar{s}}^{2}, R_{\bar{c}}^{2}$ and $C$ from the data directly, even in this Walrasian world, since we have no way to decompose the error terms $\underline{\varepsilon}_{s}$ and $\underline{\varepsilon}_{c}$ in equation (11) into $\underline{u}$ on the one hand and $\varepsilon_{s}$ and $\varepsilon_{c}$ on the other hand. $R_{w s}^{2}$ and $R_{w c}^{2}$ are therefore a lower bound for $R_{\bar{s}}^{2}$ and $R_{\bar{c}}^{2}$ respectively. The estimated coefficients $\alpha_{s}$ and $\alpha_{c}$ provide further information. The better the information on the skill variable ( $R_{\bar{s}}^{2}$ is high), the higher the coefficient $\alpha_{s}$, and mutatis mutandis the same for the complexity variable. For the special case $R_{\bar{s}}^{2}=R_{\bar{c}}^{2}$, we have $\alpha_{s}=\alpha_{c}=(1+C)^{-1}$ : the higher the correlation between the skill and complexity variable, the lower will be the coefficients $\alpha_{s}$ and $\alpha_{c}$. The intuition is that with imperfect information, $\varepsilon_{s}$ becomes a proxy for $\bar{c}$ and the other way around. If $R_{\bar{s}}^{2}=R_{\bar{c}}^{2}=1$, then $C=1$ (since Cor $[s, c]=1$ ) and the model would be unidentified by perfect collinearity of its regressors. It is tempting to give the coefficients $\alpha_{s}$ and $\alpha_{c}$ a structural interpretation. For example, referring to the old debate started by Doeringer and Piore's (1971) analysis of segmented labor markets, where wages are an attribute of jobs rather than of workers: a high value of $a_{c}$ is then interpreted as support for the segmented labor market view of the world, while a high value of $\alpha_{s}$ is interpreted as support for the traditional human 
capital interpretation. Similarly, Krueger and Summers (1988) use this type of regression to estimate inter-industry wage differentials. Those are then interpreted as evidence for non-Walrasian wage setting. The above analysis makes the simple and well known point that the regression coefficients can be just a reflection of the relative amount of unobserved heterogeneity in $s$ and $c$, and that any structural interpretation is therefore hazardous.

Can we use this framework to distinguish between the Walrasian model and the model extended with search frictions? The critical difference between both models is that in the latter log wages are positively related to the cross term $s c$, see equation (6), while in the former $s$ and $c$ are perfectly correlated and hence we are unable to establish the interaction effect of $s$ and $c$ on $\log$ wages $w$. $\mathrm{E}[w s c]$ being positive is the essence of comparative advantage: the larger $c$, the larger the effect of $s$ on $w$. The obvious way to address this question is to extend equation (11) with second order terms in the observed part of the skill and complexity indices, $\bar{s}$ and $\bar{c}$ respectively:

$$
\begin{array}{ccrccc}
\bar{w} & = & \alpha_{s} \bar{s} & + & \alpha_{c} \bar{c} & +\alpha_{s s}\left(\bar{s}^{2}-\mathrm{E}\left[\bar{s}^{2}\right]\right)+\alpha_{c c}\left(\bar{c}^{2}-\mathrm{E}\left[\bar{c}^{2}\right]\right)+\alpha_{s c}(\bar{s} \bar{c}-\mathrm{E}[\bar{s} \bar{c}])+\underline{\varepsilon} \\
R^{2} & 0.607 & 0.664 & -0.172 & -0.170 & 0.429 \\
0.4479 & (182) & (207) & (21) & (22)
\end{array}
$$

The second order terms show up highly significantly. ${ }^{10}$ The issue is whether this result is sufficient to reject the simple Walrasian model, $\bar{w}=s+\underline{u}=c+\underline{u}$, or that it can be interpreted in the same way as equation (11), such that $\bar{s}^{2}$ captures part of the effect of $\varepsilon_{c}$ and $\bar{c}^{2}$ that of $\varepsilon_{s}$. We prove a negative and a positive result. For this purpose, it is useful to define: $\vec{z} \equiv\left[\bar{s}, \varepsilon_{s}, \bar{c}, \varepsilon_{c}\right]^{\prime}$.

Proposition 4 Posit the Walrasian model $w(s, c)=s=c$ and the measurement model (9). Then, any value of $\alpha_{s c}$ can be rationalized from the correlation of $\bar{s} \bar{c}$ to $\varepsilon_{s}$ and/or $\varepsilon_{c}$.

Proof. $\quad \vec{z}$ can be linearly decomposed into four orthogonal components, $\vec{v}$, such that $w=v_{1}+v_{2}+v_{3}+v_{4}, \bar{s} \equiv v_{1}+v_{3}$, and $\bar{c} \equiv v_{2}+v_{3}$. Hence, the remaining component $v_{4}$ is orthogonal to $\bar{s}$ and $\bar{c}: E\left[v_{i} v_{4}\right]=0, i=1,3$. However, the orthogonality of the components of $\vec{v}$ does not impose any restrictions on the value of third moments, $E\left[v_{i} v_{j} v_{4}\right], i=1,2,3$, $j=1,2,3$. Therefore, any value of $\alpha_{s c}$ can be rationalized this way.

Proposition 5 requires an assumption on the joint distribution of $\bar{s}, \varepsilon_{s}, \bar{c}$, and $\varepsilon_{c}$.

\footnotetext{
${ }^{10}$ Since $\bar{s}^{2}$ and $\bar{c}^{2}$ are correlated with $\overline{s c}$, both $\bar{s}^{2}$ and $\bar{c}^{2}$ enter significantly, while $\bar{s}$ and $\bar{c}$ are constructed such that their square term yields coefficient zero, see Section 3.1.
} 
Assumption 2: $\vec{v}$ follows a multivariate distribution with third moments equal to zero.

Proposition 5 Equation (9) and Assumption 2 imply that if the Walrasian model is the true model, then $\alpha_{s s}=\alpha_{s c}=\alpha_{c c}=0$ in (12).

Proof. Consider the expression for the coefficients of an OLS regression, $\vec{\alpha}=\left[X^{\prime} X\right]^{-1} X^{\prime} \vec{y}$, where $X$ is the matrix of explanatory variables and where $\vec{y} \equiv\{\bar{w}\}$ is the vector of observed $\log$ wages. The coefficients of second order terms are different from zero only if either the first and the second order terms are correlated (the cross product of the first and second order terms in $X^{\prime} X \neq 0$ ) or the second order terms are correlated with $\bar{w}$ $\left(X^{\prime} \vec{y} \neq 0\right)$. Regarding $X^{\prime} X$ : both $s$ and $c$ are linear combinations of $\vec{z}$. Since $\vec{z}$ can be linearly decomposed in four orthogonal components, $\vec{v}$, the first and the second order terms are only correlated if the third moments of these components are different from zero, or if the cross terms $\mathrm{E}\left[v_{i} v_{j}^{2}\right] \neq 0$. However, these moments are zero, due to Assumption 2. Regarding $X^{\prime} y$ : since $\underline{u}$ is uncorrelated with anything else, a potential correlation must be due to the vector of true log wages, $w$. Under the Walrasian model, it satisfies: $w=s=c$. Hence, $w$ is a linear combination of $\vec{z}$. Then, a similar argument as for $X^{\prime} X$ establishes that $w$ is uncorrelated with the second order terms, since otherwise either a third moment or a cross term of $\vec{v}$ should be non-zero. Both are ruled out by Assumption 2.

\section{Corollary 2:}

Under Assumption 2, the inclusion of second order terms does not affect the estimated value of $\alpha_{s}$ and $\alpha_{c}$, since the second and first order terms are uncorrelated, so that the $X^{\prime} X$ matrix is block diagonal.

Proposition 4 states the negative claim that we cannot learn about the relevance of the search model from the regression equation (12) without further assumptions on the joint distribution of $\vec{z}$. Moreover, $\bar{s} \bar{c}$ can capture variation in $s$ and $c$ that is correlated to neither $\bar{s}$ nor $\bar{c}$, so that even the fact that $\alpha_{s}$ and $\alpha_{c}$ are unaffected by the introduction of the second order terms (as predicted by Corollary 2) does not imply the rejection of the Walrasian model. Proposition 5 achieves the opposite of Proposition 4. It makes the positive claim that for a quite standard distributive assumption, the second order coefficients are highly informative on the size of search frictions. Under this assumption, any deviation of $\alpha_{s c}$ from zero implies a rejection of the Walrasian model. However, 
Assumption 2 can never be fully tested. We can test whether third moments of the distribution of $\bar{s}$ and $\bar{c}$, and thereby of $v_{1}+v_{3}$ and $v_{2}+v_{3}$ are equal to zero, but we can never test the symmetry of $v_{3}$ separate of that of either $v_{1}$ or $v_{2}$, neither can we test the normality of $v_{4}$. Even the residuals from equation (11), $\underline{e}$, do not provide information, for two reasons. First, we cannot distinguish between $v_{4}$ and the measurement error in wages $\underline{u}$, so that non-normality can be attributed to either source. Second, even if $\operatorname{Var}[\underline{u}]=0$, both the Walrasian model with an asymmetric $v_{4}$ and the search model with symmetric $v_{4}$ imply that $\underline{e}$ is asymmetric.

Empirically, we do find that the coefficients $\alpha_{s}$ and $\alpha_{c}$ do not change much by the inclusion of the second order terms, see equation (11) and (12). The covariance matrix of $X$ and $\vec{y}$, including $\mathrm{E}\left[w^{3}\right]$, is shown in Table 1 . For the third moments, the t-statistics for the significance of the deviation from zero are listed underneath the covariances. ${ }^{11}$ We do not present the fourth moment because they have no effect on our estimates of the higher order terms $\left(\alpha_{s s}, \alpha_{c c}\right.$ and $\left.\alpha_{s c}\right)$. Although the covariances of the first and the second order terms are small, they are all significant. This is not surprising. Given the large number of observations ( 0.2 million), any small deviation of symmetry will be detected with high significance. We conclude that the third moments of $\bar{s}$ and $\bar{c}$ are not exactly zero but that they come close.

Table 1 Covariance matrix of $w, \bar{s}$ and $\bar{c}$ for the U.S.

\begin{tabular}{|l|cc|c|}
\hline & \multicolumn{2}{|c|}{$\bar{s}$} & \multicolumn{1}{l|}{$w$} \\
\hline $\bar{s}$ & 0.13507 & & 0.13507 \\
$\bar{c}$ & 0.08066 & 0.14611 & 0.14611 \\
$\left(\bar{s}^{2}-\mathrm{E}\left[\bar{s}^{2}\right]\right)$ & -0.00182 & -0.00240 & -0.00182 \\
& $(-4.46)$ & $(-5.66)$ & $(-4.46)$ \\
$\left(\bar{c}^{2}-\mathrm{E}\left[\bar{c}^{2}\right]\right)$ & -0.00140 & -0.00177 & -0.00177 \\
& $(-2.24)$ & $(-3.85)$ & $(-3.85)$ \\
$(\bar{s} \bar{c}-\mathrm{E}[\bar{s} \bar{c}])$ & -0.00240 & -0.00140 & 0.00126 \\
& $(-5.66)$ & $(-2.24)$ & $(1.82)$ \\
\hline
\end{tabular}

|t-values| between brackets (under the null hypothesis of joint normality of $\bar{s}, \bar{c}$, and $w$ )

The sign and relative magnitude of $\alpha_{s s}, \alpha_{s c}$, and $\alpha_{c c}$ provide further evidence for the search model and makes the interpretation of the second order terms as capturing correlations with unobserved worker and job characteristics unlikely. Although we have not yet

\footnotetext{
${ }^{11}$ The assumption that $\bar{s}$ is distributed normally yields no prediction regarding the value of $\mathrm{E}\left[\bar{s}^{2}\right]$. However, the assumption implies: $\mathrm{E}\left[\bar{s}\left(\bar{s}^{2}-\mathrm{E}\left[\bar{s}^{2}\right]\right)\right]=0$ and $\mathrm{E}\left[\left(\bar{s}^{2}-\mathrm{E}\left[\bar{s}^{2}\right]\right)^{2}\right]=2 \mathrm{E}\left[\bar{s}^{2}\right]$.
} 
derived the precise relation between the search model in equation (6) and the regression model (12) (we do this in the next section), it seems natural to assume that the signs of $w_{s s}, w_{s c}$, and $w_{c c}$ carry over to $\alpha_{s s}, \alpha_{s c}$, and $\alpha_{c c}$. These sign restrictions hold in equation (12). Similarly, equation (6) implies $w_{s c}=\left|w_{s s}+w_{c c}\right|$. One would expect that this restriction carries over to equation (12): $\alpha_{s c}=\left|\alpha_{s s}+\alpha_{c c}\right|$. By and large, this restriction holds. In Section 5, we account for the effect of the unobserved components in $s$ and $c$ on this restriction. That derivation brings our test even closer to the actual results.

As a final piece of evidence, consider Proposition 4 again. It stated that one can always construct some strange distribution of $\vec{z}$ such that the second order terms can be rationalized as capturing unobserved heterogeneity. The question is: how strange should this joint distribution be? In principle, the set of joint distributions that can generate the second order terms by unobserved heterogeneity is infinitely large. Hence, we have to impose some structure to obtain an manageable characterization. We impose the assumption that the components of $\vec{v}$ are not only orthogonal, but also independent. Consider the formula for OLS regression coefficients, $\beta=\left(X^{\prime} X\right)^{-1} X^{\prime} w$. We have seen that the third moments of the observable job and worker characteristics are about zero, $\mathrm{E}\left[\bar{s}^{3}\right] \cong \mathrm{E}\left[\bar{s}^{2} \bar{c}\right] \cong \mathrm{E}\left[\bar{s} \bar{c}^{2}\right] \cong \mathrm{E}\left[\bar{c}^{3}\right] \cong 0$, that is, $X^{\prime} X$ is close to block diagonal in the first and second order terms. This fits our conclusion that the coefficients for the first order terms are hardly affected by the inclusion of the second order terms. Hence, the coefficients of the second order terms must be due to $X^{\prime} w \neq 0$. What value of the third moments of $\vec{v}$ create the value of $X^{\prime} w$ reported in Table 1? Since $w=v_{1}+v_{2}+v_{3}+v_{4}+u$ and by the independence of the four components of $\vec{v}$, we have: $\mathrm{E}\left[w \bar{s}^{2}\right]=\mathrm{E}\left[\bar{s}^{3}\right]=\mathrm{E}\left[v_{1}^{3}\right]+\mathrm{E}\left[v_{3}^{3}\right]$, $\mathrm{E}\left[w \bar{c}^{2}\right]=\mathrm{E}\left[\bar{c}^{3}\right]=\mathrm{E}\left[v_{2}^{3}\right]+\mathrm{E}\left[v_{3}^{3}\right]$, and $\mathrm{E}[w \bar{s} \bar{c}]=\mathrm{E}\left[v_{3}^{3}\right]$. So, given the assumption of the indenpendence of the components of $\vec{v}$, the only way we can rationalize our results from unobserved heterogeneity is to assume that $v_{3}$ is skewed to right, while $v_{1}$ and $v_{2}$ are skewed to the right. The degree of skewness required to yield the moments listed in Table 1 is modest, that is, much less than the skewness generated by exponential distribution, so from that perspective, explaining the second order terms from unobserved heterogeneity 
is not inconcievable. ${ }^{12}$ However, consider the results of similar regressions for five other OECD countries reported in Table $2 .{ }^{13}$ All countries satisfy the three sign restrictions on the coefficients for the second order terms that are implied by the hypothesis that wages are concave in worker and job characteristics. Except for Germany, they all satisfy the constraint that $\alpha_{s c} \cong\left|\alpha_{s s}+\alpha_{c c}\right|$. This strongly suggests that we have come across an empirical regularity. If this regularity was due to systematic non-zero third moments in the joint distribution of $\vec{v}$, then we would expect that this regularity would also show up in the covariance of first and second order effects as reported in Table 1 for the US. In Appendix C, we present the covariance matrices for the other countries. They show no regularity. We view it as unlikely that the pattern of non-symmetric distributions differing widely across OECD economies, as reported in Appendix C, goes hand in hand with coefficients for the second order terms which are about the same. The search model provides a much more parsimonious explanation for our findings.

Table 2 Estimation results for equation (12) for various other OECD countries

\footnotetext{
${ }^{12} \mathrm{~A}$ measure of skewness is $\mathrm{E}\left[v^{3}\right] / \mathrm{E}\left[v^{2}\right]^{3 / 2}$, which is equal to 2 for the exponentional distribution. In this case, we have:

$$
\begin{aligned}
\frac{\mathrm{E}\left[v_{1}^{3}\right]}{\mathrm{E}\left[v_{1}^{2}\right]^{3 / 2}} & =\frac{\mathrm{E}\left[w \bar{s}^{2}\right]-\mathrm{E}[w \bar{s} \bar{c}]}{\left(\mathrm{E}\left[\bar{s}^{2}\right]-\mathrm{E}[\bar{s} \bar{c}]\right)^{3 / 2}}=-0.243 \\
\frac{\mathrm{E}\left[v_{2}^{1}\right]}{\mathrm{E}\left[v_{2}^{1}\right]^{3 / 2}} & =-0.181 \\
\frac{\mathrm{E}\left[v_{3}^{3}\right]}{\mathrm{E}\left[v_{3}^{2}\right]^{3 / 2}} & =\frac{\mathrm{E}[w \bar{s} \bar{c}]}{\mathrm{E}[\bar{s} \bar{c}]^{3 / 2}}=0.055
\end{aligned}
$$

${ }^{13}$ The data come from the Luxembourg Income Study (http://www.lisproject.org) which is based on the Family Budget Survey (INSEE) for France, the SOEP (DIW) for Germany, the SEP (CBS) for the Netherlands and the Family Expenditure Survey (UKDA) for the UK. For Portugal we use the Quadros de Pessoal for Portugal (Ministry of Labour and Solidarity). The samples include full time, non-farmer, private sector workers aged between 16 and 65 . We calculated $\bar{s}$ and $\bar{c}$ for each country the same way as we discussed before where $\bar{s}$ captures all the observable worker characteristics (including higher order terms) that were available and $\bar{c}$ captures all the job characteristics. In particular, the information we had on $\bar{c}$ varied considerably, i.e. industry and occupation coding varied between 2 and 4 digits.
} 


\begin{tabular}{|c|c|c|c|c|c|c|c|c|}
\hline Country & year & $\bar{s}$ & $\bar{c}$ & $\left(\bar{s}^{2}-\mathrm{E}\left[\bar{s}^{2}\right]\right)$ & $\left(\bar{c}^{2}-\mathrm{E}\left[\bar{c}^{2}\right]\right)$ & $(\bar{s} \bar{c}-\mathrm{E}[\bar{s} \bar{c}])$ & $N$ & $R^{2}$ \\
\hline France & 94 & $\begin{array}{l}0.60 \\
(32.2)\end{array}$ & $\begin{array}{l}0.61 \\
(31.8)\end{array}$ & $\begin{array}{l}-0.39 \\
(10.9)\end{array}$ & $\begin{array}{c}-0.25 \\
(5.3)\end{array}$ & $\begin{array}{l}0.62 \\
(9.0)\end{array}$ & 6052 & 0.49 \\
\hline Germany & 94 & $\begin{array}{l}0.58 \\
(13.1) \\
\end{array}$ & $\begin{array}{l}0.86 \\
(32.6) \\
\end{array}$ & $\begin{array}{c}-0.38 \\
(2.7) \\
\end{array}$ & $\begin{array}{c}-0.17 \\
(2.4) \\
\end{array}$ & $\begin{array}{l}0.17 \\
(1.2) \\
\end{array}$ & 3079 & 0.38 \\
\hline Netherlands & 94 & $\begin{array}{l}0.57 \\
(18.9)\end{array}$ & $\begin{array}{l}0.72 \\
(30.7)\end{array}$ & $\begin{array}{c}-0.32 \\
(5.8)\end{array}$ & $\begin{array}{c}-0.05 \\
(1.3)\end{array}$ & $\begin{array}{l}0.40 \\
(4.3)\end{array}$ & 2251 & 0.59 \\
\hline Portugal & 97 & $\begin{array}{c}0.66 \\
(562.0)\end{array}$ & $\begin{array}{c}0.61 \\
(522.4)\end{array}$ & $\begin{array}{l}-0.19 \\
(101.7)\end{array}$ & $\begin{array}{c}-0.11 \\
(54.1)\end{array}$ & $\begin{array}{l}0.29 \\
(27.4)\end{array}$ & $1.67 \mathrm{mln}$ & 0.53 \\
\hline UK & 86 & $\begin{array}{l}0.77 \\
(36.3)\end{array}$ & $\begin{array}{l}0.59 \\
(21.1)\end{array}$ & $\begin{array}{c}-0.40 \\
(7.2)\end{array}$ & $\begin{array}{c}-0.53 \\
(3.9)\end{array}$ & $\begin{array}{l}0.82 \\
(7.93)\end{array}$ & 4850 & 0.42 \\
\hline
\end{tabular}

\subsection{A structural interpretation in the context of the search model}

If $s$ and $c$ would be fully observed, the empirical implementation of equation (7) would be simple. We would calculate the correlation between $s$ and $c, \sqrt{\rho}$, from the data, estimate equation (6) to obtain an estimate for $\omega$ and calculate $x$ from equation (7), using a value for $\beta$ derived from the empirical literature, for example Abowd and Lemieux (1993). Given that we do not have such perfect measures, the imperfect correlation between the observed indices $\bar{s}$ and $\bar{c}$ can be due to either unobserved characteristics or search frictions which cause $s$ and $c$ themselves to be imperfectly correlated. It is useful in the context of the search model to define $C$ as the ratio of the correlation between the observed skill and complexity indices on the one hand, and the correlation between their true values on the other hand. Hence:

$$
\operatorname{Cor}[\bar{s}, \bar{c}]=\sqrt{\rho} C
$$

with $0<C<1$ : unobserved heterogeneity in $s$ and $c$ reduces their correlation. Finally, we now make the following parametric assumption:

Assumption 3: $\vec{z}$ follows a multivariate normal distribution.

Then, Proposition 6 provides a structural interpretation for the coefficients of equation (12):

Proposition 6 Consider the search model (6), and the measurement model (9) and assumption 3. Then, the regression coefficients in (12) converge to:

$$
\left[\begin{array}{c}
\alpha_{s} \\
\alpha_{c}
\end{array}\right]=\frac{1}{R_{\bar{s}}^{2} R_{\bar{c}}^{2}-\rho C^{2}}\left[\begin{array}{c}
R_{\bar{c}}^{2}\left(R_{\bar{s}}^{2}-\rho C\right) \\
R_{\bar{s}}^{2}\left(R_{\bar{c}}^{2}-C\right)
\end{array}\right]
$$


while the coefficients for the second order terms converge to:

$$
\begin{aligned}
{\left[\begin{array}{l}
\alpha_{s s} \\
\alpha_{s c} \\
\alpha_{c c}
\end{array}\right] } & =\omega(1-\rho)^{2} \frac{R_{\bar{s}}^{2} R_{\bar{c}}^{2}}{\left(R_{\bar{s}}^{2} R_{\bar{c}}^{2}-\rho C^{2}\right)^{2}}\left[\begin{array}{c}
-\rho R_{\bar{c}}^{2} C \\
\left(R_{\bar{s}}^{2} R_{\bar{c}}^{2}+\rho C^{2}\right) \\
-R_{\bar{s}}^{2} C
\end{array}\right] \\
& =\omega(1-\rho)^{2} \frac{\alpha_{s} \alpha_{c}}{\left(\alpha_{s}+\alpha_{c}-1\right)^{2}}\left[\begin{array}{c}
-\alpha_{s}\left(1-\alpha_{s}\right) \\
1+2 \alpha_{s} \alpha_{c}-\alpha_{s}-\alpha_{c} \\
-\alpha_{c}\left(1-\alpha_{c}\right)
\end{array}\right]
\end{aligned}
$$

where we apply equation (14) in the second line.

The proof is in Appendix D. Contrary to the Walrasian case, $\alpha_{s}$ and $\alpha_{c}$ are identified if both $s$ and $c$ are perfectly observed $\left(R_{\bar{s}}^{2}=R_{\bar{c}}^{2}=C=1\right)$. The reason is that $s$ and $c$ are no longer perfectly correlated in the presence of search frictions, so that there is no multicollinearity problem and the estimated coefficients converge to their true values $\alpha_{s}=w_{s}=1$ and $\alpha_{c}=w_{c}=0$ in that case.

Equation (15) reveals that the search model imposes two non-linear restrictions on the extended Mincer equation (12), relating the coefficients $\alpha_{s c} / \alpha_{s s}$ and $\alpha_{s c} / \alpha_{c c}$ to $\alpha_{s}$ and $\alpha_{c}$. As can be easily verified from the first line of equation (15), if $s$ and $c$ are perfectly observable $\left(R_{\bar{s}}^{2}=R_{\bar{c}}^{2}=C=1\right)$, then $\alpha_{c c}=-\omega$. The second line of equation (15) yields a number of testable implications of the search model. First, from Proposition 5, if there were no search frictions $(\rho=1)$, then the coefficients of the second order terms would be zero. This possibility is clearly rejected by the data. Second, $\alpha_{s c}$ is positive and the signs of $\alpha_{s s}$ and $\alpha_{c c}$ must be negative since (14) implies that $\alpha_{s}$ and $\alpha_{c}$ are between 0 and 1. These restrictions are clearly satisfied, see the estimation results for equation (12) in Section 2. A stricter tests applies the two non-linear restrictions. We estimate (10), (12) and (15) simultaneously by non-linear least squares. Because we have more than 200,000 observations, this restriction is just rejected by an F-test but the $R^{2}$ of this model and (12) is equal up to four decimals. All in all, we interpret the above as strong evidence in favor of the interpretation of the second order terms as being due to search frictions.

Regrettably, we are unable to identify $\omega$ and $\rho$ separately, since they enter in the same way in all three equations (15). We can therefore estimate $\omega(1-\rho)^{2}$, but not its two components. The intuition is that a high value of $\alpha_{s c}$ can be due to two factors. Either, the correlation between $\bar{s}$ and $\bar{c}$ is low due to large search frictions and no measurement error, leading to a high $\operatorname{Var}[c \mid s]$ and hence a low correlation $\rho$ between $s$ and $c$. But then 
$\alpha_{c c}$ is a fairly accurate estimate of $\omega$. Or, the low correlation between $\bar{s}$ and $\bar{c}$ is mainly low due to large measurement error in $s$ and $c$, so that $\operatorname{Var}[c \mid s]$ is low and $\rho$ is high. But then $\alpha_{c c}$ underestimates $\omega$ due to attenuation bias, so that the cost of a suboptimal assignment is high due to a strong curvature of $y(s, c)$. Since we cannot establish $\rho$ directly from the data, we have no way to distinguish between both stories. Alternatively, we can phrase this problem in terms of equation (3). Either, there is a lot of unobserved heterogeneity in $s$ and $c$, so that we underestimate $\left|y_{c c}\right|$ by attenuation bias, but then we overstate $\Delta^{*^{2}}$ because most of the imperfect correlation between $\bar{s}$ and $\bar{c}$ is due to unobserved heterogeneity, not to search frictions. Or, we observe $s$ and $c$ well. In that case, our estimate of $\left|y_{c c}\right|$ is reasonably accurate, but then all the imperfect correlation is due to search frictions.

The non-linear least squares estimation of (15) for the US yields (t-value between brackets):

$$
\omega(1-\rho)^{2}=0.1476
$$

The observed correlation between $\bar{s}$ and $\bar{c}$ provides a lower bound for $\rho$, where all imperfect correlation between $\bar{s}$ and $\bar{c}$ is attributed to search frictions and none to unobserved heterogeneity: $\rho>\operatorname{Cor}[\bar{s}, \bar{c}]^{2}$. Hence, equations (7), (8), (13), (14), (15) and the fact that $\operatorname{Var}[w]=0.402$ imply:

$$
\begin{aligned}
\rho & >\operatorname{Cor}[\bar{s}, \bar{c}]^{2}=0.3296 \\
\omega & =0.1476 \times(1-\rho)^{-2}>0.3285 \\
\beta x & =3 \omega \rho(1-\rho) \operatorname{Var}[w]>0.0736(\text { for } \rho<0.6704)
\end{aligned}
$$

Table 3 structural estimates (lower bounds) for other countries

\begin{tabular}{|l|l|llll|}
\hline Country & year & $\omega(1-\rho)^{2}$ & $\rho>$ & $\omega>$ & $\beta x>$ \\
\hline France & 94 & 0.1640 & 0.3633 & 0.4046 & 0.0685 \\
\hline Germany & 94 & 0.1431 & 0.1597 & 0.2027 & 0.0151 \\
\hline The Netherlands & 94 & 0.1743 & 0.2142 & 0.2823 & 0.0248 \\
\hline Portugal & 97 & 0.0869 & 0.3873 & 0.2315 & 0.0470 \\
\hline UK & 86 & 0.3969 & 0.1875 & 0.6013 & 0.0600 \\
\hline
\end{tabular}

The values for other OECD countries are given by Table 3 for $\operatorname{Var}[w]=0.4 \cong \sigma^{2}$, see equation (8). Many studies have tried to establish $\beta$ empirically. Alternatively, we 
can line up with the common practice in the search literature, and set $\beta$ equal to its "neutral" value of $1 / 2$, as we do here. Then, the complexity dispersion parameter should be $\left|y_{c c}\right| \equiv 2 \beta^{-1} \omega=4 \omega>0.30$ (see Proposition 1) and the cost of search $x>15 \%$. However, direct estimates of $\omega$ suggest much higher values than 0.30. Teulings (2005) derives a relation between the complexity dispersion parameter and Katz and Murphy's (1992) estimate of the elasticity of substitution between low and high skilled workers. This relation implies that the complexity dispersion parameter is in the order of $2,{ }^{14}$ so $\omega=0.5$ which suggests a higher value for $\rho .^{15}$ Using this value for $\omega$ and applying equation (13) and (17) (from Appendix C) yields:

$$
\begin{aligned}
\rho & =1-\sqrt{\frac{0.148}{0.500}}=0.456 \\
C & =\frac{\operatorname{Cor}[\bar{s}, \bar{c}]}{\sqrt{\rho}}=\sqrt{\frac{0.330}{0.456}}=0.851 \\
R_{\bar{s}}^{2} & =\frac{0.664}{1-0.607} \times 0.456 \times 0.851=0.656 \\
\beta x & =3 \times 0.5 \times(1-0.656) \times 0.656 \times 0.402=0.136
\end{aligned}
$$

The value of the share of observed characteristics in the total variance of $c, R_{\bar{s}}^{2}$, seems to be in line with what is known about the signal to noise ratio for in particular education data. If we take those numbers as a benchmark, the cost of search is in the order of $x \cong 27 \%$.

However, there is an alternative way to estimate $x$, not from wage data, but from unemployment. One can show that if $\beta=\frac{1}{2}$ and there is no on-the-job-search, then the cost of search are distributed evenly among its three components: the rate of unemployment, the rate of vacancies, and the cost of suboptimal assignment, see Teulings and Gautier (2004). Hence, the cost of search is three times the natural unemployment rate, that is, $x \cong 3 \times 5 \%=15 \%$, about half as high as the estimate based on wage data.

Can we reconcile these two independent and conflicting pieces of evidence? In Gautier,

\footnotetext{
${ }^{14}$ This might even be a conservative estimate of the complexity dispersion parameter, since it assumes that demand for the output of various job types $c$ is governed by a Leontieff technology. Hence, changes in the assignment of workers to jobs are the only source of substitutability between worker types. A more flexible technology than Leontieff would shift part of the substitutability to the demand for job types $c$, thereby reducing the amount of substitution due to the assingment process, hence raising $\omega$.

${ }^{15}\left|y_{c c}\right|=\left(\eta_{\text {low-high }} \operatorname{Var}[w]\right)^{-1}=(1.4 \times 0.40)^{-1} \simeq 2$. This relation provides a lower bound for $\omega$ since it assumes a Leontieff technology in the demand for the output of various $c$-types. Allowing for substitution between $c$-types yields higher values of $\omega$.
} 
Teulings and van Vuuren (2005), we construct an assignment model with on the job search. In the model with on the job search, the reservation wage is smaller than the value of search while unemployed because workers no longer give up the full option value of search, since they can continue search on the job. We therefore define $x$ as the difference between $y^{*}(s)$ and the flow value of unemployment. This extension narrows the gap for two reasons. First, $y^{*}(s)$ remains the same but the value of unemployment increases when introducing on the job search because wages now contain a "no quit" premium. Secondly, $\mathrm{E}[\Delta c]$ becomes smaller because workers keep moving to the diagonal $c(s)$ in Figure $1 .^{16}$

\section{Final remarks}

We conclude this paper by relating our results to the two strands in the literature that have been discussed in Section 1. First, our results have implications for the discussion on interindustry wage differentials initiated by Krueger and Summers' (1988) classic paper. The framework laid out in Section 2 basically points at a fundamental problem of interpreting the results of wage regressions including both worker and job characteristics in the set of regressors: the one will be a proxy for the unobserved component of the other and vice versa. This insight is all but new. Since Krueger and Summers (1988), many papers have addressed this issue by using panel data, initially to control for unobserved worker characteristics, and more recently to control for unobserved worker and job characteristics simultaneously, by using matched firm-worker data, see Abowd, Kramarz, and Margolis (1998). The debate has not yet been settled.

The contribution of this paper is to show that the set up in Krueger and Summers might be mistaken. Their regressions suggest that some industries are universally "better" than others, as they pay higher wages. This feature is due to the additivity of their log wage function in worker and job characteristics. Hence, their wage function is not log supermodular, as is required for comparative advantage. In a world with comparative advantage / log supermodularity there is no such thing as a universally "better" job. The wage for a worker of a particular type is concave in the characteristics of the job she holds, and there is an interior maximum. A "higher" job type than this "optimal" job type yields a lower wage. Furthermore, the "optimal" job type depends on a worker's characteristics. In our regressions, we allow for this concavity by entering second order

\footnotetext{
${ }^{16}$ The relation between $x$ and unemployment becomes $-2 u \ln u$ which equals 0.30 for $u$ is 0.05 .
} 
terms in worker and job characteristics. These turn out to be highly significant, which puts into question the interpretation of Krueger and Summers' industry dummies as capturing efficiency wage effects or rents. In models without frictions, the fact that we observe positive assortative matching is typically explained by comparative advantage of high skilled workers in complex jobs and this view seems to be uncontroversial. There is no reason to abandon this assumption when frictions are introduced. Similarly, our results have implications for the methodology of Abowd, Kramarz, and Margolis (1998). Interestingly, while unobserved characteristics deem hopeless any attempt to provide a structural interpretation of the relative magnitude of the coefficients for worker and job characteristics in a cross section analysis, we have shown that the second order terms are much less sensitive to this problem. One must make quite extreme assumptions on the distribution of the error terms to rationalize these coefficients by unobserved heterogeneity. We provide some simple formulas to correct the coefficients of the second order terms for the effect of unobserved heterogeneity assuming their distribution to be normal.

Second, a comparison of our approach to the literature on the estimation of assignment models offers an alternative interpretation for what is at stake. Rosen's (1974) seminal paper on hedonic pricing and assignment sparkled a debate on what variation is required for identification of the underlying production and utility functions in this type of model. In terms of this paper, how can we identify the curvature of $y(s, c)$ ? As pointed out by Heckman and Sedlacek (1985), following early contributions by Roy (1951), identification is problematic because people self-select into the job type $c$ that yields the highest output in a Walrasian equilibrium. In their models with only two job types, there is sufficient within job variation in $s$ left to identify a large part of $y(s, c)$, after correcting for the selectivity of worker types by using standard techniques. In our model, which is essentially a continuous version of the Roy model, this strategy no longer works. The equilibrium assignment is characterized by a one-to-one correspondence of $s$ to $c$, denoted $s(c)$. This one-to-one correspondence yields a perfect correlation between $s$ and $c$, which renders any attempt to estimate the full functional form of $y(s, c)$ hopeless by a standard multicollinearity problem. One can establish $y[s, c(s)]=y^{*}(s)$ (from the zero profit condition $w^{*}(s)=y^{*}(s)$ ) and one can establish its first derivative $y_{s}[s, c(s)]$ (from the first order condition for optimal assignment, $\left.w^{*^{\prime}}(s)=y_{s}[s, c(s)]\right)$, but not its curvature $y_{s s}[s, c(s)]$. In a Walrasian equilibrium, we observe $y(s, c)$ only for its optimal assignment $c=c(s)$, 
and not for other values of $c$. Kahn and Lang (1988) suggested to use variation between markets in the distribution of the supply of $s$ or the demand for $c$. This leads to different equilibrium assignments $c(s)$ in various markets, which allows for the identification of $y_{s s}(s, c)$. Ekeland, Heckman, and Nesheim (2004) exploit the generic non-linearity of the equilibrium assignment $c(s)$.

Here, we travel another route. Workers cannot afford to search for ever for an optimal job when search is costly. They are forced to accept jobs at which they produce less than the maximum output, that is, $c \neq c(s)$. This process breaks down the perfect correlation between $s$ and $c$ that characterizes the Walrasian equilibrium. Obviously, we have information on log wages $w(s, c)$ and not on log output $y(s, c)$. However, when we assume that gains from a better match quality are shared in some fixed way between the worker and the firm, the curvature in wages is informative on the curvature in output, see Figure 2. Adding second order terms in the appropriately transformed indices, $s$ and $c$ allows us to estimate this curvature in wages. Regrettably, the formulas to correct the coefficients for the effect of measurement error do not allow for a complete identification of the underlying structure. They only provide sensible lower bounds for the importance of search frictions. The formulas imply that the output losses due to search frictions are in the order of $25 \%$, which is substantial.

\section{References}

Abowd, J.M. F. Kramarz, D.N. Margolis (1998) High wage workers and high wage firms, Econometrica, 67, 251-333.

Abowd, J.M. F. Kramarz, P. Lengermann, S. Perez-Duarte (2004) Are Good Workers Employed by Good Firms? A test of a simple assortative mating model for France and the United States," mimeo Cornell.

Abowd, J.M. And T. Lemieux (1993) The effects of product market competition on collective bargaining agreements: the case of foreign competition in Canada, Quarterly Journal of Economics, 108, 983-1014.

Angrist J. And A.B. Krueger (1991) Does Compulsory School Attendance Affect Schooling and Earnings?, Quarterly Journal of Economics, 1991, 106, 979-1014

Becker, G. (1973) A Theory of Marriage: Part I, Journal of Political Economy, 81 813-46. 
Bound J. And A. Krueger (1991) The Extent of Measurement Error in Longitudinal Earnings Data: Do two wrongs make a right?, Journal of Labor Economics, 9, 1-24.

Burdett K. And D. Mortensen (1998) Equilibrium wage differentials and employer size, International Economic Review, 39, 257-274.

Dickens W.T. And L. Katz (1987) Inter -Industry wage differences and theories of wage determination, NBER working paper N0. 2271.

Doeringer P. And M. Piore (1971), Internal Labor Markets and Manpower Analysis, M.E. Sharpe

Ekeland, I., J.J. Heckman, and L. Nesheim, (2004) Identification and Estimation of Hedonic Models, Journal of Political Economy, 112, S60-S109.

Gautier, P.A. C.N. Teulings and A. van Vuuren (2005) On the job search and sorting, Tinbergen Institute discussion paper.

Heckman, J.J., And G. Sedlacek, (1985) Heterogeneity, Aggregation, and Market Wage Functions: An Empirical Model of Self-Selection in the Labor Market, Journal of Political Economy, 93, 1077-1125.

Jovanovic B. (1979) Job matching and the theory of turnover, Journal of Political Economy, 87, 972-990.

Katz, L.F. And K.M. MurPhy (1992) Changes in relative wages, 1963-1987: supply and demand factors, Quarterly Journal of Economics, 107, 35-78.

Kahn, S. and K. Lang. (1988) Efficient Estimation of Structural Hedonic Systems, International Economic Review, 29, 157-66.

Krueger A.B. And L.H. Summers (1988), Efficiency wages and the inter-industry wage structure, Econometrica, 259-93.

Murphy, K. And R.H. Topel (1987) Unemployment, risk, and earnings: testing for equalizing differences in the labor market, in: K. Lang and J.S. Leonard, eds., Unemployment and the structure of labor markets, 103-140. Oxford: Basil Blackwell.

Mortensen D.T. (2002) How Are (Danish) Wages Determined?, mimeo Northwestern University

Pissarides C.A. (2000) Equilibrium unemployment theory, 2nd edition, MIT press., Cambridge MA. 
Postel Vinay and J.M. Robin (2002) Equilibrium Wage Dispersion with Worker and Employer Heterogeneity, Econometrica, 70, 2295-2330.

Rosen, S. (1974) Hedonic prices and implicit markets: product differentiation in pure competition", Journal of Political Economy , 82, 34-55.

Roy, A. (1951) Some thoughts on the distribution of earnings, Oxford Economic Papers, 3, 135-146.

Sattinger M. (1975) Comparative advantage and the distribution of earnings and abilities, Econometrica, 43, 455-68.

Shimer R. And L. Smith (2000) Assortative matching and search, Econometrica, 68, 343-370.

Teulings C.N. (1995) The wage distribution in a model of the assignment of skills to jobs, Journal of Political Economy, 280-315.

Teulings C.N. (2005) Comparative advantage, relative wages, and the accumulation of human capital, Journal of Political Economy. Forthcoming.

Teulings C.N. And P.A. Gautier (2002) Search and the city, Tinbergen Institute discussion paper $061 / 3$.

Teulings C.N. And P.A. Gautier (2004) The right man for the job, Review of Economic Studies, 71, 2, 553-580.

VAn DEn Berg G. And G. Ridder (1998) An empirical equilibrium search model of the labor market, Econometrica, 66, 1183-1221

\section{Appendix}

\section{A Proof of Proposition 1}

The normalizations of $w, s$ and $c$ imply:

$$
\begin{aligned}
& \mathrm{E}[\mathrm{E}[w(s, c) \mid s]]=\mathrm{E}[s]=\mathrm{E}[w]=0 \\
& \mathrm{E}[\mathrm{E}[w(s, c) \mid c]]=\mathrm{E}[c]=\mathrm{E}[w]=0
\end{aligned}
$$

Hence, since $\mathrm{E}[\mathrm{E}[c \mid s]]=\mathrm{E}[c]=0$ :

$$
\mathrm{E}[\mathrm{E}[c \mid s]]=\mathrm{E}\left[\rho_{0}+\rho s\right]=\rho_{0}=0
$$


and by the same argument $\tau_{0}=0$. Hence, we can write:

$$
\begin{aligned}
& s=\tau c+\Delta s \\
& c=\rho s+\Delta c
\end{aligned}
$$

where $\mathrm{E}[\Delta c]=\mathrm{E}[\Delta s]=\operatorname{Cov}[c, \Delta s]=\operatorname{Cov}[s, \Delta c]=0$. By the definition of $c(s)$ we have:

$$
\begin{aligned}
w_{c}[s, c(s)] & =w_{c}+w_{s c} s+w_{c c} c(s)=0 \Rightarrow \\
c(s) & =-\frac{w_{c}+w_{s c} s}{w_{c c}}
\end{aligned}
$$

Hence, $c(s)$ is a linear function of $s$. Therefore $\mathrm{E}[c(s)]=c[\mathrm{E}(s)]=c(0)$. Since $c(s)=\mathrm{E}[c \mid s], \mathrm{E}[c(s)]=\mathrm{E}[\mathrm{E}[c|s|]]=\mathrm{E}[c]=0$, we have $c(0)=0$. This implies $w_{c}=0$. Combining these results yields:

$$
\begin{aligned}
w(s, c) & =w_{0}+w_{s} s+\frac{1}{2} w_{s s} s^{2}+w_{s c} s c+\frac{1}{2} w_{c c} c^{2} \\
& =w_{0}+w_{s} s+\frac{1}{2} w_{s s} s^{2}+w_{s c} s(\rho s+\Delta c)+\frac{1}{2} w_{c c}(\rho s+\Delta c)^{2} \\
& =w_{0}+w_{s}(\tau c+\Delta s)+\frac{1}{2} w_{s s}(\tau c+\Delta s)^{2}+w_{s c}(\tau c+\Delta s) c+\frac{1}{2} w_{c c} c^{2} \\
& \left.\Rightarrow \begin{array}{c}
d^{2} \mathrm{E}[w(s, c) \mid s] / d s^{2}=w_{s s}+2 \rho w_{s c}+\rho^{2} w_{c c} \equiv 0 \\
d^{2} \mathrm{E}[w(s, c) \mid c] / d c^{2}=\tau^{2} w_{s s}+2 \tau w_{s c}+w_{c c} \equiv 0
\end{array}\right\} \\
& \left.\Rightarrow \begin{array}{c}
\mathrm{d}[w(s, c) \mid s] / d s=w_{s} \equiv 1 \\
w_{s c}[w(s, c) \mid c] / d c=\tau w_{s} \equiv 1
\end{array}\right\} \Rightarrow \tau=1 \Rightarrow \begin{array}{c}
w_{s s}=-2 \rho \omega \\
w_{s}[w
\end{array}
\end{aligned}
$$

where $\omega \equiv-\frac{1}{2} w_{c c}$ and $\sigma^{2} \equiv \operatorname{Var}[s]$. Hence, $\operatorname{Cov}[s, c]=\mathrm{E}[s(\rho s+\Delta c)]=\rho \sigma^{2}=\mathrm{E}[c(c+\Delta s)]=\mathrm{E}\left[c^{2}\right]$, $\operatorname{Var}[c]=\rho \sigma^{2}$ and $\mathrm{E}\left[\Delta c^{2}\right] \cong \rho(1-\rho) \sigma^{2}$. Q.E.D.

\section{A.1 Second moment of $w$}

The variance of $w$ is derived from Proposition 1:

$$
\begin{aligned}
w & =w_{0}+s-\omega\left[-\rho s^{2}+(1+\rho) s(\rho s+\Delta c)-(\rho s+\Delta c)^{2}\right] \\
& =w_{0}+s-\omega\left[(1+\rho) s \Delta c-\Delta c^{2}\right]
\end{aligned}
$$

using an expression for $w_{0}$ (which can be derived from the relation $\mathrm{E}[w]=0$ ) and $\operatorname{Var}[s]=$ $\sigma^{2}, \operatorname{Var}[\Delta c]=\rho(1-\rho) \sigma^{2}, \mathrm{E}\left[\Delta c^{3}\right] \cong 0$ (by its symmetry around $\mathrm{E}[\Delta c]$ ) and $\mathrm{E}\left[\Delta c^{4}\right] \cong$ $3 \operatorname{Var}[\Delta c]^{2}$ (taking the ratio between $4^{\text {th }}$ and $2^{\text {th }}$ moment for the normal distribution):

$$
\operatorname{Var}[w] \simeq \sigma^{2}+\rho(1-\rho)^{2}(1+\rho) \sigma^{4} \omega^{2}
$$




\section{B Proof of Proposition 3}

Define: $X_{1} \equiv[s, c]$ be the matrix of 'true' first order effects. Accordingly, let $\bar{X}_{1}$ denote the 'observed' first order effects. Since all variables are measured in deviation from their mean, we can ignore the intercept. By the definitions in Section 2, the moments of the sub-matrices $\bar{X}_{1}^{\prime} \bar{X}_{1}$ and $\bar{X}_{1}^{\prime} y$ read:

$$
\begin{aligned}
\frac{1}{N} \mathrm{E}\left[\bar{X}_{1}^{\prime} w\right] & =\sigma^{2}\left[\begin{array}{l}
R_{\bar{s}}^{2} \\
R_{\bar{c}}^{2}
\end{array}\right] \\
\frac{1}{N} \mathrm{E}\left[\bar{X}_{1}^{\prime} \bar{X}_{1}\right] & =\sigma^{2}\left[\begin{array}{cc}
R_{\bar{s}}^{2} & C \\
C & R_{\bar{c}}^{2}
\end{array}\right]
\end{aligned}
$$

where $N$ is the number of observations in the regression. Consider equation (11). Applying the expression for OLS coefficients proves the Proposition. Q.E.D.

\section{Covariance matrices for various countries}

Table 4 Standard errors under joint normality

\begin{tabular}{|l|ll|}
\hline & $\bar{s}$ & $\bar{c}$ \\
$\left(\bar{s}^{2}-\mathrm{E}\left[\bar{s}^{2}\right]\right)$ & $\sigma_{s}^{3} \sqrt{15 / N}$ & $\sigma_{s}^{2} \sigma_{c}^{1} \sqrt{15 / N}$ \\
$\left(\bar{c}^{2}-\mathrm{E}\left[\bar{c}^{2}\right]\right)$ & $\sigma_{c}^{2} \sigma_{s}^{1} \sqrt{15 / N}$ & $\sigma_{c}^{3} \sqrt{15 / N}$ \\
$(\bar{s} \bar{c}-\mathrm{E}[\bar{s} \bar{c}])$ & $\sigma_{s}^{2} \sigma_{c}^{1} \sqrt{15 / N}$ & $\sigma_{c}^{2} \sigma_{s}^{1} \sqrt{15 / N}$ \\
\hline
\end{tabular}

Table 6 Covariance of $w, \bar{s}$ and $\bar{c}$ for France $(N=6052)$

\begin{tabular}{|l|rl|r|}
\hline & \multicolumn{1}{|c|}{$\bar{s}$} & \multicolumn{1}{l|}{$w$} \\
\hline $\bar{s}$ & 0.11813 & & 0.11813 \\
$\bar{c}$ & 0.06835 & 0.10884 & 0.10884 \\
$\left(\bar{s}^{2}-\mathrm{E}\left[\bar{s}^{2}\right]\right)$ & -0.00480 & 0.00884 & -0.00488 \\
$\left(\bar{c}^{2}-\mathrm{E}\left[\bar{c}^{2}\right]\right)$ & 0.01316 & 0.01775 & 0.01775 \\
$(\bar{s} \bar{c}-\mathrm{E}[\bar{s} \bar{c}])$ & 0.00884 & 0.01316 & 0.01349 \\
\hline
\end{tabular}

Table 7 Covariance of $w, \bar{s}$ and $\bar{c}$ for Germany $(N=3079)$ 


\begin{tabular}{|l|ll|l|}
\hline & $\bar{s}$ & $\bar{c}$ & $w$ \\
\hline $\bar{s}$ & 0.03530 & & 0.03530 \\
$\bar{c}$ & 0.02061 & 0.07537 & 0.07537 \\
$\left(\bar{s}^{2}-\mathrm{E}\left[\bar{s}^{2}\right]\right)$ & 0.00674 & 0.00531 & 0.00694 \\
$\left(\bar{c}^{2}-\mathrm{E}\left[\bar{c}^{2}\right]\right)$ & 0.00584 & 0.00383 & 0.00383 \\
$(\bar{s} \bar{c}-\mathrm{E}[\bar{s} \bar{c}])$ & 0.00531 & 0.00584 & 0.00709 \\
\hline
\end{tabular}

Table 8 Covariance of $w, \bar{s}$ and $\bar{c}$ for the Netherlands $(N=2251)$

\begin{tabular}{|l|rr|r|}
\hline & \multicolumn{2}{|c|}{$\bar{c}$} & \multicolumn{1}{l|}{} \\
\hline $\bar{s}$ & 0.08610 & & 0.08610 \\
$\bar{c}$ & 0.04102 & 0.09123 & 0.09123 \\
$\left(\bar{s}^{2}-\mathrm{E}\left[\bar{s}^{2}\right]\right)$ & -0.03252 & -0.01064 & -0.03188 \\
$\left(\bar{c}^{2}-\mathrm{E}\left[\bar{c}^{2}\right]\right)$ & -0.00181 & -0.01071 & -0.00107 \\
$(\bar{s} \bar{c}-\mathrm{E}[\bar{s} \bar{c}])$ & -0.01064 & -0.00181 & -0.00743 \\
\hline
\end{tabular}

Table 9 Covariance of $w, \bar{s}$ and $\bar{c}$ for Portugal $(N=1671267)$

\begin{tabular}{|l|ll|l|}
\hline & $\bar{s}$ & $\bar{c}$ & $w$ \\
\hline $\bar{s}$ & 0.14724 & & 0.14725 \\
$\bar{c}$ & 0.10670 & 0.17873 & 0.17873 \\
$\left(\bar{s}^{2}-\mathrm{E}\left[\bar{s}^{2}\right]\right)$ & 0.06703 & 0.04905 & 0.06703 \\
$\left(\bar{c}^{2}-\mathrm{E}\left[\bar{c}^{2}\right]\right)$ & 0.05534 & 0.08872 & 0.08872 \\
$(\bar{s} \bar{c}-\mathrm{E}[\bar{s} \bar{c}])$ & 0.04905 & 0.05534 & 0.06325 \\
\hline
\end{tabular}

Table 10 Covariance of $w, \bar{s}$ and $\bar{c}$ for the $\mathrm{UK}(N=4850)$

\begin{tabular}{|l|rl|r|}
\hline & \multicolumn{1}{|c|}{$\bar{s}$} & \multicolumn{1}{l|}{$w$} \\
\hline $\bar{s}$ & 0.09208 & & 0.09208 \\
$\bar{c}$ & 0.03166 & 0.05806 & 0.05806 \\
$\left(\bar{s}^{2}-\mathrm{E}\left[\bar{s}^{2}\right]\right)$ & -0.00658 & 0.00194 & -0.00658 \\
$\left(\bar{c}^{2}-\mathrm{E}\left[\bar{c}^{2}\right]\right)$ & 0.00341 & 0.00514 & 0.00514 \\
$(\bar{s} \bar{c}-\mathrm{E}[\bar{s} \bar{c}])$ & 0.00194 & 0.00341 & 0.00493 \\
\hline
\end{tabular}

\section{Proof of Proposition 6}

Previous definitions imply the following covariance matrix of $\bar{s}, s, \bar{c}$, and $c$ :

$$
\operatorname{Var}[\bar{s}, \bar{c}, s, c]=\sigma^{2}\left[\begin{array}{cccc}
R_{\bar{s}}^{2} & \rho C & R_{\bar{s}}^{2} & \rho R_{\bar{s}}^{2} \\
\rho C & \rho R_{\bar{c}}^{2} & \rho R_{\bar{c}}^{2} & \rho R_{\bar{c}}^{2} \\
R_{\bar{s}}^{2} & \rho R_{\bar{c}}^{2} & 1 & \rho \\
\rho R_{\bar{s}}^{2} & \rho R_{\bar{c}}^{2} & \rho & \rho
\end{array}\right]
$$


Analogous to $X_{1}$ and $\bar{X}_{1}$, let $X_{2} \equiv\left[s-E\left[s^{2}\right], s c-E[s c], c-E[c]\right]$, the covariance matrix of 'true' second order effects and let $\bar{X}_{2}$ denote the covariance matrix of 'observed' second order effects, both net of their mean. Since all variables are considered in deviation from their mean, we can ignore the intercept. By Assumption $2, \bar{X}_{1}^{\prime} \bar{X}_{2}=0$, so that the $X^{\prime} X$ matrix for equation (12) is block diagonal. Hence, we can invert the sub-matrices $\bar{X}_{1}^{\prime} \bar{X}_{1}$ and $\bar{X}_{2}^{\prime} \bar{X}_{2}$ separately. Hence, the first and second order terms can be derived independently.

First, consider the first order terms. $\bar{X}_{1}^{\prime} \bar{X}_{1}$ can be taken from equation (16). Since second order terms are uncorrelated to first order terms, only the term $s$ in equation (6) is correlated to $\bar{X}_{1}$. Hence: $\mathrm{E}\left[\bar{X}_{1}^{\prime} w\right]=\mathrm{E}\left[\bar{X}_{1}^{\prime} s\right]$, which can again be taken from equation (16). Applying the expression for OLS coefficients yields:

$$
\left[\begin{array}{c}
\alpha_{s} \\
\alpha_{c}
\end{array}\right]=\left[\begin{array}{cc}
R_{\bar{s}}^{2} & \rho C \\
\rho C & \rho R_{\bar{c}}^{2}
\end{array}\right]^{-1}\left[\begin{array}{c}
R_{\bar{s}}^{2} \\
\rho R_{\bar{c}}^{2}
\end{array}\right]=\frac{1}{R_{\bar{s}}^{2} R_{\bar{c}}^{2}-\rho C^{2}}\left[\begin{array}{c}
R_{\bar{c}}^{2}\left(R_{\bar{s}}^{2}-\rho C\right) \\
R_{\bar{s}}^{2}\left(R_{\bar{c}}^{2}-C\right)
\end{array}\right]
$$

Rearranging terms gives:

$$
\begin{aligned}
\frac{C}{R_{\bar{c}}^{2}} & =\frac{1-\alpha_{c}}{\alpha_{s}} \\
\frac{\rho C}{R_{\bar{s}}^{2}} & =\frac{1-\alpha_{s}}{\alpha_{c}}
\end{aligned}
$$

Next, consider the second order terms. Define: $w_{2} \equiv \omega[-\rho, 1+\rho,-1]^{\prime}$ be the vector of coefficients of the 'true' second order effects. Only the second order terms in equation 6) are correlated to $\bar{X}_{2}$. These second order terms read: $X_{2} w_{2}$. Hence: $\mathrm{E}\left[\bar{X}_{2}^{\prime} w\right]=\mathrm{E}\left[\bar{X}_{2}^{\prime} X_{2}\right] w_{2}$. The moments of the sub-matrices $\bar{X}_{2}^{\prime} \bar{X}_{2}$ and $\bar{X}_{2}^{\prime} X_{2}$ read: ${ }^{17}$

$$
\begin{aligned}
& \frac{1}{N} \mathrm{E}\left[\bar{X}_{2}^{\prime} X_{2}\right]=\sigma^{4}\left[\begin{array}{ccc}
2 R_{\bar{s}}^{4} & 2 \rho R_{\bar{s}}^{4} & 2 \rho^{2} R_{\bar{s}}^{4} \\
2 \rho R_{\bar{s}}^{2} R_{\bar{c}}^{2} & \left(\rho+\rho^{2}\right) R_{\bar{s}}^{2} R_{\bar{c}}^{2} & 2 \rho^{2} R_{\bar{s}}^{2} R_{\bar{c}}^{2} \\
2 \rho^{2} R_{\bar{c}}^{4} & 2 \rho^{2} R_{\bar{c}}^{4} & 2 \rho^{2} R_{\bar{c}}^{4}
\end{array}\right] \\
& \frac{1}{N} \mathrm{E}\left[\bar{X}_{2}^{\prime} \bar{X}_{2}\right]=\sigma^{4}\left[\begin{array}{ccc}
2 R_{\bar{s}}^{4} & 2 \rho R_{\bar{s}}^{2} C & 2 \rho^{2} C^{2} \\
2 \rho R_{\bar{s}}^{2} C & \rho R_{\bar{s}}^{2} R_{\bar{c}}^{2}+\rho^{2} C^{2} & 2 \rho^{2} R_{\bar{c}}^{2} C \\
2 \rho^{2} C^{2} & 2 \rho^{2} R_{\bar{c}}^{2} C & 2 \rho^{2} R_{\bar{c}}^{4}
\end{array}\right]
\end{aligned}
$$

\footnotetext{
${ }^{17}$ We use the fourth moment of the multivariate normal distribution:

$\mathrm{E}\left[x_{1}^{4}\right]=3 \sigma_{1}^{4} \Rightarrow \mathrm{E}\left[x_{1}^{4}\right]-\mathrm{E}\left[x_{1}^{2}\right]^{2}=2 \sigma_{1}^{4}$

$\mathrm{E}\left[x_{1}^{3} x_{2}\right]=3 \sigma_{1}^{2} \sigma_{12} \Rightarrow \mathrm{E}\left[x_{1}^{3} x_{2}\right]-\mathrm{E}\left[x_{1}^{2}\right] \mathrm{E}\left[x_{1} x_{2}\right]=2 \sigma_{1}^{2} \sigma_{12}$

$\mathrm{E}\left[x_{1}^{2} x_{2}^{2}\right]=\sigma_{1}^{2} \sigma_{2}^{2}+2 \sigma_{12}^{2}$

$\Rightarrow \mathrm{E}\left[x_{1}^{2} x_{2}^{2}\right]-\mathrm{E}\left[x_{1}^{2}\right] \mathrm{E}\left[x_{2}^{2}\right]=2 \sigma_{12}^{2}$

$\Rightarrow \mathrm{E}\left[x_{1}^{2} x_{2}^{2}\right]-\mathrm{E}\left[x_{1} x_{2}\right]^{2}=\sigma_{1}^{2} \sigma_{2}^{2}+\sigma_{12}^{2}$

$\mathrm{E}\left[x_{1} x_{2} x_{3}^{2}\right]-\mathrm{E}\left[x_{1} x_{2}\right] \mathrm{E}\left[x_{3}^{2}\right]=2 \sigma_{13} \sigma_{23}$

$\mathrm{E}\left[x_{1} x_{2} x_{3} x_{4}\right]-\mathrm{E}\left[x_{1} x_{2}\right] \mathrm{E}\left[x_{3} x_{4}\right]=\sigma_{13} \sigma_{24}+\sigma_{14} \sigma_{23}$.

with $x_{1}, x_{2}, x_{3}, x_{4}$ being a zero mean multivariate normal
} 
Define: $\alpha_{2} \equiv\left[\alpha_{s s}, \alpha_{s c}, \alpha_{c c}\right]$. Then:

$$
\begin{aligned}
\alpha_{2} & =\operatorname{plim}\left[\left(\bar{X}_{2}^{\prime} \bar{X}_{2}\right)^{-1} \bar{X}_{2}^{\prime} w\right]=\operatorname{plim}\left[\left(\bar{X}_{2}^{\prime} \bar{X}_{2}\right)^{-1} \bar{X}_{2}^{\prime} X_{2}\right] w_{2} \\
& =\omega(1-\rho)^{2} \frac{R_{\bar{s}}^{2} R_{\bar{c}}^{2}}{\left(R_{\bar{s}}^{2} R_{\bar{c}}^{2}-\rho C^{2}\right)^{2}}\left[\begin{array}{c}
-\rho R_{\bar{c}}^{2} C \\
\left(R_{\bar{s}}^{2} R_{\bar{c}}^{2}+\rho C^{2}\right) \\
-R_{\bar{s}}^{2} C
\end{array}\right]
\end{aligned}
$$

Q.E.D.

\section{E Figures}
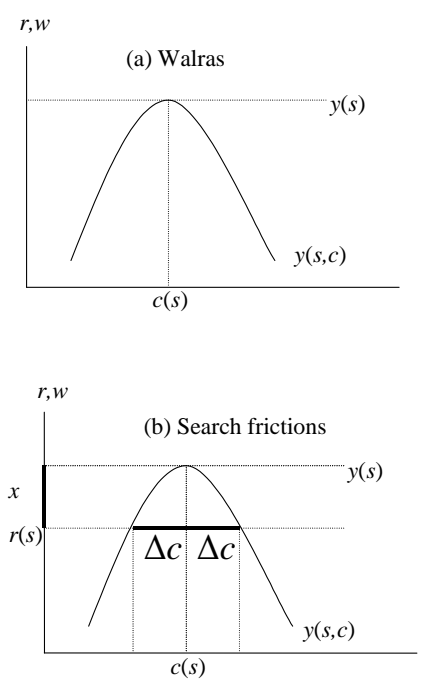


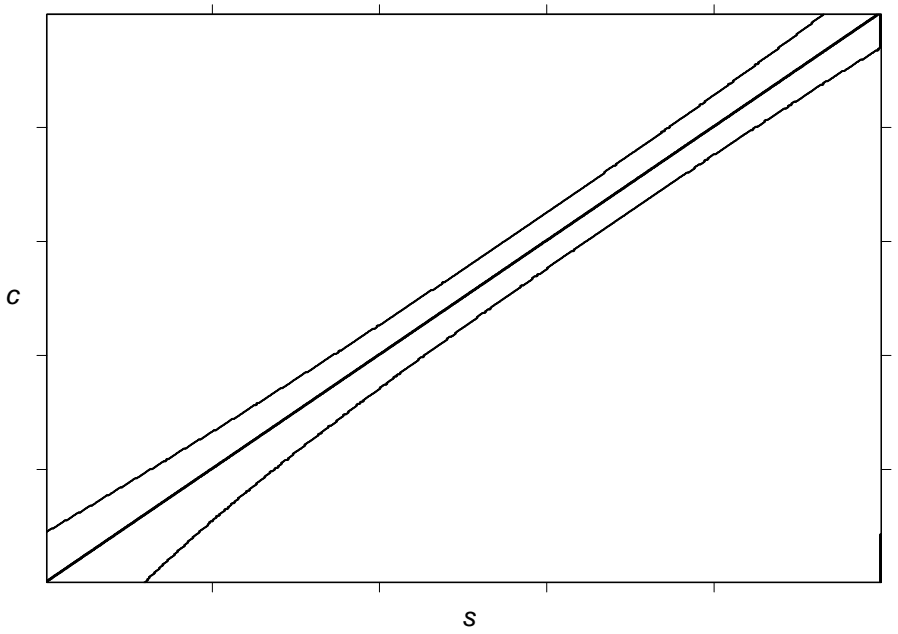

Figure 1: The aggregate search equilibrium
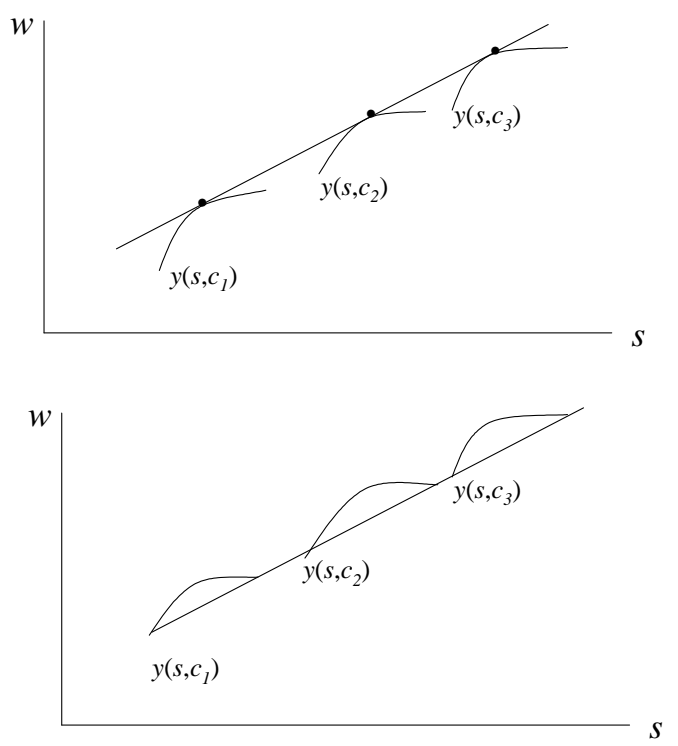

Figure 2: Identification with and without search frictions 